El ect ri c- di pol e, el ect ri c- quadr upol e, magnet i c- di pol e, and magnet i c- quadr upol e transiti ons in the neon i soel ect roni $c$ sequence

\begin{tabular}{|c|c|}
\hline $\begin{array}{l}\text { j our nal or } \\
\text { publ i cat } i \text { on } \mathrm{title}\end{array}$ & PHYSI CAL REVI EW A \\
\hline vol une & Vol . 64 \\
\hline nunber & I ssue 1 \\
\hline page $r$ ange & $012507-1-012507-13$ \\
\hline year & 2001- 06- 01 \\
\hline URL & ht t p: //hdl . handl e. net /10655/323 \\
\hline
\end{tabular}




\title{
Electric-dipole, electric-quadrupole, magnetic-dipole, and magnetic-quadrupole transitions in the neon isoelectronic sequence
}

\author{
U. I. Safronova, ${ }^{1,2}$ C. Namba, ${ }^{1}$ I. Murakami, ${ }^{1}$ W. R. Johnson, ${ }^{2}$ and M. S. Safronova ${ }^{2}$ \\ ${ }^{1}$ National Institute for Fusion Science, Toki, Gifu, 509-5292, Japan \\ ${ }^{2}$ Department of Physics, University of Notre Dame, Notre Dame, Indiana 46556
}

(Received 27 February 2001; published 15 June 2001)

\begin{abstract}
Excitation energies for $2 l-3 l^{\prime}$ hole-particle states of Ne-like ions are determined to second order in relativistic many-body perturbation theory (MBPT). Reduced matrix elements, line strengths, and transition rates are calculated for electric-dipole $(E 1)$, magnetic-quadrupole $(E 2)$, magnetic-dipole $(M 1)$, and magneticquadrupole $(M 2)$ transitions in Ne-like ions with nuclear charges ranging from $Z=11$ to 100 . The calculations start from a $1 s^{2} 2 s^{2} 2 p^{6}$ closed-shell Dirac-Fock potential and include second-order Coulomb and BreitCoulomb interactions. First-order many-body perturbation theory (MBPT) is used to obtain intermediatecoupling coefficients, and second-order MBPT is used to determine the matrix elements. Contributions from negative-energy states are included in the second-order $E 1, M 1, E 2$, and $M 2$ matrix elements. The resulting transition energies are compared with experimental values and with results from other recent calculations. Trends of $E 1, E 2, M 1$, and $M 2$ transition rates as functions of nuclear charge $Z$ are shown graphically for all transitions to the ground state.
\end{abstract}

DOI: 10.1103/PhysRevA.64.012507

PACS number(s): 32.70.Cs, 31.25.Jf, 31.15.Md

\section{INTRODUCTION}

Excitation energies, line strengths, and transition probabilities for $2 s^{2} 2 p^{5} 3 l$ and $2 s 2 p^{6} 3 l$ states along the neon isoelectronic sequence have been studied theoretically and experimentally during the past 30-40 years. Z-expansion [1-3], model potential [4-7], configuration-interaction [8-12], multiconfiguration Hartree-Fock [13-15], $R$-matrix [16], multiconfiguration Dirac-Fock (MCDF) [17], and relativistic many-body perturbation theory (MBPT) [18-20] are among the methods that have been used to calculate these quantities for Ne-like ions.

Nonrelativistic perturbation theory was used in Refs. [1-3] to calculate energy levels of $2 s^{2} 2 p^{5} 3 l$ and $2 s 2 p^{6} 3 l$ states in Ne-like ions. In those papers, contributions from the Coulomb and Breit interactions were represented in powers of $1 / Z$. Accurate transition energies and rates were obtained for ions with nuclear charges $Z$ from 20 to 60 , by introducing screening constants and including radiative and higher-order relativistic effects. The technique used in Refs. [1-3] is referred to as the $M Z$ method. Results obtained by the $M Z$ method and two versions of the model potential method [4-7] were compared in Ref. [21] for the $2 l-3 l^{\prime}$ electricdipole transition energies in Ne-like ions with $Z=36-92$. Correlation corrections for Ne-like ions were studied empirically by Quinet $e t$ al. in Ref. [17], where uncorrected MCDF energies were given and differences between the experimental and uncorrected MCDF energies were treated by leastsquares fitting. The resulting adjusted energies for $2 s^{2} 2 p^{5} 3 l$ states were given for ions with $Z=28-92$. A similar idea was used by Hibbert et al. [11], where accurate adjusted energies for $2 s^{2} 2 p^{5} 3 l$ and $2 s 2 p^{6} 3 l$ states were presented for ions with $Z=10-36$. At a more sophisticated level, relativistic MBPT was used by Avgoustouglou and co-workers in Refs. [19,20], to determine energies of four $2 s^{2} 2 p^{5} 3 s$ levels and three $2 s^{2} 2 p^{5} 3 d$ levels for ions with $Z=10-92$, where second-order MBPT for the Coulomb and Breit correlation corrections was supplemented by all-order hole-core corrections.

Accurate measurements along the Ne isoelectronic sequence have been made using a variety of light sources. Most accurate wavelength measurements come from plasma light sources, both magnetic-fusion plasmas and laserproduced plasmas. Early wavelength measurements for high$Z$ ions were made by Aglitskii et al. [2], Boiko et al. [22], Gordon and co-workers [23,24], Jupén and Litzén [25], Gauthier et al. [26], Beiersdorfer et al. [27], and Buchet et al. [28]. Some of the work was summarized by Aglitskii et al. [21] in a report on measurements of $\mathrm{Kr}^{26+}-\mathrm{Nd}^{50+}$ spectra observed in low-inductance vacuum spark-produced plasmas [21]. Beiersdorfer et al. [29] reported results in Ne-like $\mathrm{Xe}, \mathrm{La}, \mathrm{Nd}$, and $\mathrm{Eu}$, obtained at the Princeton Tokamak. Some years later, Beiersdorfer and co-workers [30,31] presented spectra of $\mathrm{Ne}-$ like $\mathrm{Yb}$ and $\mathrm{Th}$ obtained in an electronbeam ion trap (EBIT). Beam-foil spectra of $2 l-3 l^{\prime}$ transitions in highly-stripped gold and bismuth were reported by Chandler et al. [32] and Dietrich et al. [33]. Recently, accurate wavelength measurements of lasing and nonlasing lines in a laser-produced germanium plasma were reported by Yuan et al. in Ref. [34]. Wavelengths of x-ray transitions in $\mathrm{Ne}$-like $\mathrm{I}^{43+}, \mathrm{Cs}^{45+}$, and $\mathrm{Ba}^{46+}$ were measured with a flat crystal spectrometer at the Tokyo EBIT by Nakamura et al. in Ref. [35].

In the present paper, MBPT is used to determine energies of $2 s^{2} 2 p^{5} 3 l(J)$ and $2 s 2 p^{6} 3 l(J)$ states of Ne-like ions with nuclear charges $Z=11-100$. Energies are calculated for the 16 even-parity excited states and the 20 odd-parity excited states. The calculations are carried out to second order in perturbation theory. Corrections for the frequency-dependent Breit interaction are included in first order only. Lamb shift corrections to energies are also estimated and included.

Relativistic MBPT is used to determine reduced matrix 
elements, oscillator strengths, and transition rates for all allowed and forbidden electric-dipole and magnetic-dipole ( $E 1$ and $M 1)$ and electric-quadrupole and magneticquadrupole transitions ( $E 2$ and $M 2)$ from $2 s^{2} 2 p^{5} 3 l(J)$ and $2 s 2 p^{6} 3 l(J)$ excited states into the ground state in Ne-like ions. Retarded $E 1$ and $E 2$ matrix elements are evaluated in both length and velocity forms. The present MBPT calculations start from a nonlocal $1 s^{2} 2 s^{2} 2 p^{6}$ Dirac-Fock potential, and consequently give gauge-dependent transition matrix elements. Second-order correlation corrections compensate almost exactly for the gauge dependence of the first-order matrix elements, leading to corrected matrix elements that differ by less than $1 \%$ in length and velocity forms throughout the $\mathrm{Ne}$ isoelectronic sequence.

\section{METHOD}

Details of the MBPT method were presented in Refs. [36-38] for a calculation of energies and $E 1$ and $M 1$ radiative transition rates in two-particle states and in Ref. [39] for calculation of energies and $E 1$ oscillator strengths of holeparticle states. We apply the formulas given in Ref. [39] for calculation of energies and $E 1$ transition rates, and extend those formulas to obtain $M 1, E 2$, and $M 2$ transition rates in $\mathrm{Ne}$-like systems. In this section, we present first- and secondorder contributions for the transition energies and retarded $E 1, M 1, E 2$, and $M 2$ matrix elements in Ne-like ions. We refer the reader to Refs. [36-39] for detailed expressions for the various contributions to these quantities. The calculations are carried out using sets of basis Dirac-Hartree-Fock (DHF) orbitals. The orbitals used in the present calculation are obtained as linear combinations of $B$ splines. These $B$-spline basis orbitals are determined using the method described in Ref. [40]. We use $40 B$ splines of order 8 for each singleparticle angular momentum state, and we include all orbitals with orbital angular momentum $l \leqslant 7$ in our basis set.

\section{A. Model space}

The model space for $2 s^{2} 2 p^{5} 3 l(J)$ and $2 s 2 p^{6} 3 l(J)$ states of Ne-like ions is formed from particle-hole states of the type $a_{v}^{+} a_{a}|0\rangle$, where $|0\rangle$ is the closed-shell $1 s_{1 / 2}^{2} 2 s_{1 / 2}^{2} 2 p_{1 / 2}^{2} 2 p_{3 / 2}^{4}$ ground state. For our study of low-lying $2 l^{-1} 3 l^{\prime}$ states of Ne-like ions, values of $a$ are $2 s_{1 / 2}, 2 p_{1 / 2}$, and $2 p_{3 / 2}$, while values of $v$ are $3 s_{1 / 2}, 3 p_{1 / 2}, 3 p_{3 / 2}, 3 d_{3 / 2}$, and $3 d_{5 / 2}$. Combining the $n=2$ hole orbitals and the $n=3$ particle orbitals in Ne-like ions, we obtain 20 odd-parity states consisting of three $J=0$ states, seven $J=1$ states, six $J=2$ states, three $J=3$ states, and one $J=4$ state. Additionally, there are 16 even-parity states consisting of three $J=0$ states, six $J=1$ states, five $J=2$ states, and two $J=3$ states. The distribution of the 36 states in the model space is summarized in Table I. In this table, we give both $j j$ and $L S$ designations for holeparticle states. Instead of using the $2 l_{j}^{-1} 3 l_{j^{\prime}}^{\prime}$ or $2 l^{-1} 3 l^{\prime}$ designations, we use simpler designations $2 l_{j} 3 l_{j^{\prime}}^{\prime}$ or $2 l 3 l^{\prime}(J)$ in this table. The same $\left(2 l 3 l^{\prime}\right)$ designations are used for simplicity in all following tables and the text below.
TABLE I. Possible hole-particle states in the $2 l_{j} 3 l^{\prime}{ }_{j^{\prime}}$ complexes; $j j$ and $L S$ coupling schemes.

\begin{tabular}{|c|c|c|c|}
\hline \multicolumn{2}{|c|}{ Even-parity states } & \multicolumn{2}{|c|}{ Odd-parity states } \\
\hline$j j$ coupling & $L S$ coupling & $j j$ coupling & $L S$ coupling \\
\hline $2 p_{3 / 2} 3 p_{3 / 2} \quad(0)$ & $2 p 3 p^{3} P_{0}$ & $2 p_{1 / 2} 3 s_{1 / 2}$ & $2 p 3 s^{3} P_{0}$ \\
\hline $2 p_{1 / 2} 3 p_{1 / 2}$ & $2 p 3 p{ }^{1} S_{0}$ & $2 p_{3 / 2} 3 d_{3 / 2}(0)$ & $2 p 3 d^{3} P_{0}$ \\
\hline $2 s_{1 / 2} 3 s_{1 / 2}$ & $2 s 3 s^{1} S_{0}$ & $2 s_{1 / 2} 3 p_{1 / 2}$ & $2 s 3 p^{3} P_{0}$ \\
\hline $2 p_{3 / 2} 3 p_{1 / 2}$ & $2 p 3 p^{3} S_{1}$ & $2 p_{3 / 2} 3 s_{1 / 2}$ & $2 p 3 s^{3} P_{1}$ \\
\hline $2 p_{3 / 2} 3 p_{3 / 2}$ & $2 p 3 p^{3} D_{1}$ & $2 p_{1 / 2} 3 s_{1 / 2}$ & $2 p 3 s{ }^{1} P_{1}$ \\
\hline $2 p_{1 / 2} 3 p_{1 / 2}$ & $2 p 3 p{ }^{1} P_{1}$ & $2 p_{3 / 2} 3 d_{3 / 2}$ & $2 p 3 d^{3} P_{1}$ \\
\hline $2 p_{1 / 2} 3 p_{3 / 2}$ & $2 p 3 p^{3} P_{1}$ & $2 p_{3 / 2} 3 d_{5 / 2}$ & $2 p 3 d^{3} D_{1}$ \\
\hline $2 s_{1 / 2} 3 s_{1 / 2}$ & $2 s 3 s^{3} S_{1}$ & $2 p_{1 / 2} 3 d_{3 / 2}$ & $2 p 3 d^{1} P_{1}$ \\
\hline \multirow[t]{2}{*}{$2 s_{1 / 2} 3 d_{3 / 2}$} & $2 s 3 d^{3} D_{1}$ & $2 s_{1 / 2} 3 p_{1 / 2}$ & $2 s 3 p^{3} P_{1}$ \\
\hline & & $2 s_{1 / 2} 3 p_{3 / 2}$ & $2 s 3 p{ }^{1} P_{1}$ \\
\hline $2 p_{3 / 2} 3 p_{1 / 2}$ & $2 p 3 p^{3} D_{2}$ & $2 p_{3 / 2} 3 s_{1 / 2}$ & $2 p 3 s{ }^{3} P_{2}$ \\
\hline $2 p_{3 / 2} 3 p_{3 / 2}$ & $2 p 3 p^{3} P_{2}$ & $2 p_{3 / 2} 3 d_{5 / 2}$ & $2 p 3 d^{3} P_{2}$ \\
\hline $2 p_{1 / 2} 3 p_{3 / 2}$ (2) & $2 p 3 p{ }^{1} D_{2}$ & $2 p_{3 / 2} 3 d_{3 / 2}$ & $2 p 3 d^{3} F_{2}$ \\
\hline $2 s_{1 / 2} 3 d_{3 / 2}$ & $2 s 3 d^{3} D_{2}$ & $2 p_{1 / 2} 3 d_{3 / 2}$ & $2 p 3 d^{1} D_{2}$ \\
\hline \multirow[t]{2}{*}{$2 s_{1 / 2} 3 d_{5 / 2}$} & $2 s 3 d{ }^{1} D_{2}$ & $2 p_{1 / 2} 3 d_{5 / 2}$ & $2 p 3 d^{3} D_{2}$ \\
\hline & & $2 s_{1 / 2} 3 p_{3 / 2}$ & $2 s 3 p^{3} P_{2}$ \\
\hline $2 p_{3 / 2} 3 p_{3 / 2}$ & $2 p 3 p{ }^{3} D_{3}$ & $2 p_{3 / 2} 3 d_{3 / 2}$ & $2 p 3 d^{3} F_{3}$ \\
\hline \multirow[t]{3}{*}{$2 s_{1 / 2} 3 d_{5 / 2}$} & $2 s 3 d^{3} D_{3}$ & $2 p_{3 / 2} 3 d_{5 / 2}$ & $2 p 3 d^{3} D_{3}$ \\
\hline & & $2 p_{1 / 2} 3 d_{5 / 2}$ & $2 p 3 d^{1} F_{3}$ \\
\hline & & $2 p_{3 / 2} 3 d_{5 / 2}$ & $2 p 3 d^{3} F_{4}$ \\
\hline
\end{tabular}

\section{B. Example: energy matrix for $\mathrm{Mo}^{32+}$}

In Table II, we give various contributions to the secondorder energies for the special case of Ne-like molybdenum, $Z=42$. In this table, we show the one- and two-body secondorder Coulomb contributions to the energy matrix labeled $E_{1}^{(2)}$ and $E_{2}^{(2)}$, respectively. The corresponding BreitCoulomb contributions are given in columns headed $B_{1}^{(2)}$ and $B_{2}^{(2)}$ of Table II. The one-body second-order energy is obtained as a sum of the valence $E_{v}^{(2)}$ and hole $E_{a}^{(2)}$ energies, with the latter being the dominant contribution. The values of $E_{1}^{(2)}$ and $B_{1}^{(2)}$ are nonzero only for diagonal matrix elements. Although there are 36 diagonal and 142 nondiagonal matrix elements for $\left(2 l 3 l^{\prime}\right)(J)$ hole-particle states in $j j$ coupling, we list only the subset with $J=0$ in Table II. Both even- and odd-parity states are presented. It can be seen from the table that second-order Breit-Coulomb corrections are relatively large and, therefore, must be included in accurate calculations. The values of nondiagonal matrix elements given in columns headed $E_{2}^{(2)}$ and $B_{2}^{(2)}$ are comparable with values of diagonal two-body matrix elements. However, the values of one-body contributions, $E_{1}^{(2)}$ and $B_{1}^{(2)}$, are larger than the values of two-body contributions, $E_{2}^{(2)}$ and $B_{2}^{(2)}$, respectively. As a result, total second-order diagonal matrix elements are much larger than the nondiagonal matrix elements, which are shown in Table III.

In Table III, we present results for the zeroth-, first-, and second-order Coulomb contributions, $E^{(0)}, E^{(1)}$, and $E^{(2)}$, and the first- and second-order Breit-Coulomb corrections, $B^{(1)}$ and $B^{(2)}$. It should be noted that corrections for the 
TABLE II. Second-order contributions to the energy matrices (a.u.) for even- and odd-parity states with $J=0$ in the case of Ne-like molybdenum, $Z=42$. One- and two-body second-order Coulomb and BreitCoulomb contributions are given in columns labeled $E_{1}^{(2)}, E_{2}^{(2)}, B_{1}^{(2)}$, and $B_{2}^{(2)}$, respectively.

\begin{tabular}{|c|c|c|c|c|}
\hline \multirow[b]{2}{*}{$2 l_{1} j_{1} 3 l_{2} j_{2}, 2 l_{3} j_{3} 3 l_{4} j_{4}$} & \multicolumn{2}{|c|}{ (a) Coulomb interaction } & \multicolumn{2}{|c|}{ (b) Breit-Coulomb correction } \\
\hline & $E_{1}^{(2)}$ & $E_{2}^{(2)}$ & $B_{1}^{(2)}$ & $B_{2}^{(2)}$ \\
\hline \multicolumn{5}{|c|}{ Even-parity states, $J=0$} \\
\hline $2 p_{3 / 2} 3 p_{3 / 2}, 2 p_{3 / 2} 3 p_{3 / 2}$ & -0.093121 & -0.021694 & 0.023048 & 0.000666 \\
\hline $2 p_{1 / 2} 3 p_{1 / 2}, 2 p_{1 / 2} 3 p_{1 / 2}$ & -0.104719 & -0.004136 & 0.023404 & 0.002373 \\
\hline $2 s_{1 / 2} 3 s_{1 / 2}, 2 s_{1 / 2} 3 s_{1 / 2}$ & -0.137890 & -0.010115 & 0.018779 & 0.001201 \\
\hline $2 p_{3 / 2} 3 p_{3 / 2}, 2 p_{1 / 2} 3 p_{1 / 2}$ & 0 & 0.027015 & 0 & 0.001622 \\
\hline $2 p_{1 / 2} 3 p_{1 / 2}, 2 p_{3 / 2} 3 p_{3 / 2}$ & 0 & 0.026980 & 0 & 0.001518 \\
\hline $2 p_{3 / 2} 3 p_{3 / 2}, 2 s_{1 / 2} 3 s_{1 / 2}$ & 0 & 0.000692 & 0 & -0.000444 \\
\hline $2 s_{1 / 2} 3 s_{1 / 2}, 2 p_{3 / 2} 3 p_{3 / 2}$ & 0 & 0.000541 & 0 & -0.000327 \\
\hline $2 p_{1 / 2} 3 p_{1 / 2}, 2 s_{1 / 2} 3 s_{1 / 2}$ & 0 & -0.000528 & 0 & 0.000571 \\
\hline $2 s_{1 / 2} 3 s_{1 / 2}, 2 p_{1 / 2} 3 p_{1 / 2}$ & 0 & -0.000985 & 0 & 0.000624 \\
\hline \multicolumn{5}{|c|}{ Odd-parity states, $J=0$} \\
\hline $2 p_{1 / 2} 3 s_{1 / 2}, 2 p_{1 / 2} 3 s_{1 / 2}$ & -0.097297 & 0.007555 & 0.024669 & 0.002590 \\
\hline $2 p_{3 / 2} 3 d_{3 / 2}, 2 p_{3 / 2} 3 d_{3 / 2}$ & -0.098824 & -0.005758 & 0.023456 & 0.004385 \\
\hline $2 s_{1 / 2} 3 p_{1 / 2}, 2 s_{1 / 2} 3 p_{1 / 2}$ & -0.145320 & 0.003364 & 0.017514 & 0.003414 \\
\hline $2 p_{1 / 2} 3 s_{1 / 2}, 2 p_{3 / 2} 3 d_{3 / 2}$ & 0 & 0.003797 & 0 & -0.000034 \\
\hline $2 p_{3 / 2} 3 d_{3 / 2}, 2 p_{1 / 2} 3 s_{1 / 2}$ & 0 & 0.003868 & 0 & -0.000150 \\
\hline $2 p_{1 / 2} 3 s_{1 / 2}, 2 s_{1 / 2} 3 p_{1 / 2}$ & 0 & -0.009976 & 0 & -0.000284 \\
\hline $2 s_{1 / 2} 3 p_{1 / 2}, 2 p_{1 / 2} 3 s_{1 / 2}$ & 0 & -0.007312 & 0 & -0.000382 \\
\hline $2 p_{3 / 2} 3 d_{3 / 2}, 2 s_{1 / 2} 3 p_{1 / 2}$ & 0 & -0.018302 & 0 & -0.000842 \\
\hline $2 s_{1 / 2} 3 p_{1 / 2}, 2 p_{3 / 2} 3 d_{3 / 2}$ & 0 & -0.018404 & 0 & -0.001147 \\
\hline
\end{tabular}

frequency-dependent Breit interaction [41] are included in the first order only. The difference between the first-order Breit-Coulomb corrections calculated with and without frequency dependence is less than $1 \%$. As one can see from Table III, the ratio of nondiagonal and diagonal matrix elements is larger for the first-order contributions than for the second-order contributions. Another difference in the firstand second-order contributions is the symmetry properties: the first-order nondiagonal matrix elements are symmetric, and the second-order nondiagonal matrix elements are not symmetric. The values of $E^{(2)}\left[a^{\prime} v^{\prime}(J), a v(J)\right]$ and $E^{(2)}\left[a v(J), a^{\prime} v^{\prime}(J)\right]$ matrix elements differ in some cases by a factor 2-3, and occasionally have opposite signs.

We now discuss how the final energy levels are obtained from the above contributions. To determine the first-order energies of the states under consideration, we diagonalize the symmetric first-order effective Hamiltonian, including both the Coulomb and Breit interactions. The first-order expansion coefficient $C^{N}[a v(J)]$ is the $N$ th eigenvector of the first-order effective Hamiltonian, and $E^{(1)}[N]$ is the corresponding eigenvalue. The resulting eigenvectors are used to determine the second-order Coulomb correction $E^{(2)}[N]$, the second-order Breit-Coulomb correction $B^{(2)}[N]$, and the QED correction $E_{\text {Lamb }}[N]$.

In Table IV, we list the following contributions to the energies of 36 excited states in $\mathrm{Mo}^{32+}$ : the sum of the zerothand first-order energies $E^{(0+1)}=E^{(0)}+E^{(1)}+B^{(1)}$, the second-order Coulomb energy $E^{(2)}$, the second-order Breit-
Coulomb correction $B^{(2)}$, the QED correction $E_{\mathrm{Lamb}}$, and the sum of the above contributions, $E_{\text {tot }}$. The QED correction is approximated as the sum of the one-electron self-energy and the first-order vacuum-polarization energy. The self-energy contribution is estimated for $s, p_{1 / 2}$, and $p_{3 / 2}$ orbitals by interpolating the values obtained by Mohr [42] using Coulomb wave functions. For this purpose, an effective nuclear charge $Z_{\text {eff }}$ is obtained by finding the value of $Z_{\text {eff }}$ required to give a Coulomb orbital with the same average $\langle r\rangle$ as the DHF orbital.

When starting calculations from relativistic DHF wave functions, it is natural to use $j j$ designations for uncoupled transition and energy matrix elements; however, neither $j j$ nor $L S$ coupling describes the physical states properly, except for the single-configuration state $2 p_{3 / 2} 3 d_{5 / 2}(4)$ $\equiv 2 p 3 d^{3} F_{4}$. Both designations are used in Table IV.

\section{C. $Z$ dependence of eigenvectors and eigenvalues in Ne-like ions}

In Figs. 1 and 2, we show the $Z$ dependence of the eigenvectors and eigenvalues of the $2 l j 3 l^{\prime} j^{\prime}(J)$ states for the example of odd-parity states with $J=1$. We refer to a set of states of the same parity and the same $J$ as a complex of states. This particular $J=1$ odd-parity complex includes seven states which are listed in Table I. Using the first-order expansion coefficients $C^{N}[a v(J)]$ defined in Sec. II B, we can present the resulting eigenvectors as 
TABLE III. Contributions to energy matrices (a.u.) for even- and odd-parity states with $J=0$ before diagonalization in the case of Ne-like molybdenum, $Z=42$.

\begin{tabular}{|c|c|c|c|c|c|}
\hline $2 l_{1} j_{1} 3 l_{2} j_{2}, 2 l_{3} j_{3} 3 l_{4} j_{4}$ & $E^{(0)}$ & $E^{(1)}$ & $B^{(1)}$ & $E^{(2)}$ & $B^{(2)}$ \\
\hline \multicolumn{6}{|c|}{ Even-parity states, $J=0$} \\
\hline $2 p_{3 / 2} 3 p_{3 / 2}, 2 p_{3 / 2} 3 p_{3 / 2}$ & 94.31412 & -2.14875 & -0.11363 & -0.11482 & 0.02371 \\
\hline $2 p_{1 / 2} 3 p_{1 / 2}, 2 p_{1 / 2} 3 p_{1 / 2}$ & 97.42414 & -2.69622 & -0.17075 & -0.10885 & 0.02578 \\
\hline $2 s_{1 / 2} 3 s_{1 / 2}, 2 s_{1 / 2} 3 s_{1 / 2}$ & 102.94272 & -2.60244 & -0.10796 & -0.14801 & 0.01998 \\
\hline $2 p_{3 / 2} 3 p_{3 / 2}, 2 p_{1 / 2} 3 p_{1 / 2}$ & 0 & -0.72929 & -0.00262 & 0.02702 & 0.00162 \\
\hline $2 p_{1 / 2} 3 p_{1 / 2}, 2 p_{3 / 2} 3 p_{3 / 2}$ & 0 & -0.72929 & -0.00262 & 0.02698 & 0.00152 \\
\hline $2 p_{3 / 2} 3 p_{3 / 2}, 2 s_{1 / 2} 3 s_{1 / 2}$ & 0 & 0.18869 & 0.00465 & 0.00069 & -0.00044 \\
\hline $2 s_{1 / 2} 3 s_{1 / 2}, 2 p_{3 / 2} 3 p_{3 / 2}$ & 0 & 0.18869 & 0.00465 & 0.00054 & -0.00033 \\
\hline $2 p_{1 / 2} 3 p_{1 / 2}, 2 s_{1 / 2} 3 s_{1 / 2}$ & 0 & -0.14649 & -0.00145 & 0.00053 & 0.00057 \\
\hline $2 s_{1 / 2} 3 s_{1 / 2}, 2 p_{1 / 2} 3 p_{1 / 2}$ & 0 & -0.14649 & -0.00145 & 0.00098 & 0.00062 \\
\hline \multicolumn{6}{|c|}{ Odd-parity states, $J=0$} \\
\hline $2 p_{1 / 2} 3 s_{1 / 2}, 2 p_{1 / 2} 3 s_{1 / 2}$ & 94.83453 & -3.21316 & -0.19463 & -0.08974 & 0.02726 \\
\hline $2 p_{3 / 2} 3 d_{3 / 2}, 2 p_{3 / 2} 3 d_{3 / 2}$ & 97.71474 & -3.87933 & -0.11784 & -0.10458 & 0.02784 \\
\hline $2 s_{1 / 2} 3 p_{1 / 2}, 2 s_{1 / 2} 3 p_{1 / 2}$ & 105.53232 & -3.27937 & -0.08679 & -0.14196 & 0.02093 \\
\hline $2 p_{1 / 2} 3 s_{1 / 2}, 2 p_{3 / 2} 3 d_{3 / 2}$ & 0 & -0.05389 & 0.00241 & 0.00380 & -0.00003 \\
\hline $2 p_{3 / 2} 3 d_{3 / 2}, 2 p_{1 / 2} 3 s_{1 / 2}$ & 0 & -0.05389 & 0.00241 & 0.00387 & -0.00015 \\
\hline $2 p_{1 / 2} 3 s_{1 / 2}, 2 s_{1 / 2} 3 p_{1 / 2}$ & 0 & 0.43065 & 0.00145 & -0.00998 & -0.00028 \\
\hline $2 s_{1 / 2} 3 p_{1 / 2}, 2 p_{1 / 2} 3 s_{1 / 2}$ & 0 & 0.43065 & 0.00145 & -0.00731 & -0.00038 \\
\hline $2 p_{3 / 2} 3 d_{3 / 2}, 2 s_{1 / 2} 3 p_{1 / 2}$ & 0 & 0.43608 & -0.00470 & -0.01830 & -0.00084 \\
\hline $2 s_{1 / 2} 3 p_{1 / 2}, 2 p_{3 / 2} 3 d_{3 / 2}$ & 0 & 0.43608 & -0.00470 & -0.01840 & -0.00115 \\
\hline \multicolumn{6}{|c|}{$\Phi(N)=C^{N}\left[2 p_{3 / 2} 3 s_{1 / 2}(1)\right] \Phi\left[2 p_{3 / 2} 3 s_{1 / 2}(1)\right]+C^{N}\left[2 p_{1 / 2} 3 s_{1 / 2}(1)\right] \Phi\left[2 p_{1 / 2} 3 s_{1 / 2}(1)\right]$} \\
\hline \multicolumn{6}{|c|}{$+C^{N}\left[2 p_{3 / 2} 3 d_{3 / 2}(1)\right] \Phi\left[2 p_{3 / 2} 3 d_{3 / 2}(1)\right]+C^{N}\left[2 p_{3 / 2} 3 d_{5 / 2}(1)\right] \Phi\left[2 p_{3 / 2} 3 d_{5 / 2}(1)\right]$} \\
\hline \multicolumn{6}{|c|}{$+C^{N}\left[2 p_{1 / 2} 3 d_{3 / 2}(1)\right] \Phi\left[2 p_{1 / 2} 3 d_{3 / 2}(1)\right]+C^{N}\left[2 s_{1 / 2} 3 p_{1 / 2}(1)\right] \Phi\left[2 s_{1 / 2} 3 p_{1 / 2}(1)\right]$} \\
\hline \multicolumn{6}{|c|}{$+C^{N}\left[2 s_{1 / 2} 3 p_{3 / 2}(1)\right] \Phi\left[2 s_{1 / 2} 3 p_{3 / 2}(1)\right]$} \\
\hline
\end{tabular}

As a result, $49 C^{N}[a v(J)]$ coefficients are needed to describe the seven eigenvalues. These coefficients are often called mixing coefficients. For simplicity, we plot only four of the mixing coefficients for the level $N=2 p 3 d^{3} D_{1}$ in Fig. 1. These coefficients are chosen to illustrate the mixing of the states; the remaining mixing coefficients give very small contributions to this level. We observe strong mixing between $2 p_{1 / 2} 3 s_{1 / 2}(1)$ and $2 p_{3 / 2} 3 d_{5 / 2}(1)$ states for $Z=54-55$, which results in the dramatic changes in their values.

Energies, relative to the ground state, of odd-parity states with $J=1$, divided by $(Z-7)^{2}$, are shown in Fig. 2 . We plot the four energy levels within the odd-parity $J=1$ complex which are involved in the mixing of states illustrated in Fig. 1. We use $L S$ designations for small $Z$ and $j j$ designations for large $Z$ in the figure. It should be noted that the $L S$ designations are chosen by comparing our results with available experimental data for low- $Z$ ions. It was already shown in Fig. 1 that the largest mixing coefficient contributing to the $2 p 3 d^{3} D_{1}$ level is not the same for $Z \leqslant 54$ and $Z \geqslant 55$. This change is seen in Fig. 2. The first and third curves almost cross for $Z=55$; the difference in energy values at
$Z=55$ is equal to just $0.27 \%$ of the total-energy values. Strong mixing also occurs for other levels, for example, between $\left[2 p_{1 / 2} 3 d_{3 / 2}(1)\right]$ and $\left[2 s_{1 / 2} 3 p_{1 / 2}(1)\right]$ states for $Z$ $=69-70$, and between $\left[2 p_{3 / 2} 3 d_{5 / 2}(2)\right]$ and $\left[2 p_{3 / 2} 3 d_{3 / 2}(2)\right]$ states for $Z=36-37$.

\section{Electric-dipole and electric-quadrupole matrix elements}

We calculate electric-dipole $(E 1)$ matrix elements for the transitions between the seven odd-parity $2 s_{1 / 2} 3 p_{j}(1)$, $2 p_{j} 3 s_{1 / 2}(1)$, and $2 p_{j} 3 d_{j^{\prime}}(1)$ excited states and the ground state and electric-quadrupole $(E 2)$ matrix elements between the five even-parity $2 p_{j} 3 p_{j^{\prime}}(2)$ and $2 s_{1 / 2} 3 d_{j}(2)$ excited states and the ground state for Ne-like ions with nuclear charges $Z=11-100$. The evaluation of the reduced $E 1$ matrix elements for neonlike ions follows the corresponding calculation for nickel-like ions described in Ref. [39]. The calculation of the reduced $E 2$ matrix elements is carried out using the same MBPT formulas given in Ref. [39], and replacing the one-particle $E 1$ matrix elements by the oneparticle $E 2$ matrix elements given in Ref. [43]. The first- and 
TABLE IV. Energies (a.u.) of Ne-like molybdenum, $Z=42$, relative to the ground state. $E^{(0+1)} \equiv E^{(0)}$ $+E^{(1)}+B^{(1)}$.

\begin{tabular}{|c|c|c|c|c|c|c|}
\hline$L S$ coupling & $j j$ coupling & $E^{(0+1)}$ & $E^{(2)}$ & $B^{(2)}$ & $E_{\text {Lamb }}$ & $E_{\text {tot }}$ \\
\hline $2 p 3 p^{3} P_{0}$ & $2 p_{3 / 2} 3 p_{3 / 2}(0)$ & 91.8510 & -0.1012 & 0.0246 & -0.0047 & 91.7698 \\
\hline $2 p 3 p{ }^{1} S_{0}$ & $2 p_{1 / 2} 3 p_{1 / 2}(0)$ & 94.7484 & -0.1225 & 0.0249 & 0.0006 & 94.6514 \\
\hline $2 s 3 s^{1} S_{0}$ & $2 s_{1 / 2} 3 s_{1 / 2}(0)$ & 100.2417 & -0.1480 & 0.0199 & -0.0674 & 100.0462 \\
\hline $2 p 3 p^{3} S_{1}$ & $2 p_{3 / 2} 3 p_{1 / 2}(1)$ & 89.7840 & -0.0860 & 0.0270 & -0.0068 & 89.7182 \\
\hline $2 p 3 p^{3} D_{1}$ & $2 p_{3 / 2} 3 p_{3 / 2}(1)$ & 90.8239 & -0.0899 & 0.0258 & -0.0052 & 90.7545 \\
\hline $2 p 3 p{ }^{1} P_{1}$ & $2 p_{1 / 2} 3 p_{1 / 2}(1)$ & 93.8402 & -0.0956 & 0.0271 & 0.0010 & 93.7728 \\
\hline $2 p 3 p^{3} P_{1}$ & $2 p_{1 / 2} 3 p_{3 / 2}(1)$ & 94.8700 & -0.0968 & 0.0267 & 0.0023 & 94.8022 \\
\hline $2 s 3 s^{3} S_{1}$ & $2 s_{1 / 2} 3 s_{1 / 2}(1)$ & 99.7699 & -0.1338 & 0.0207 & -0.0667 & 99.5900 \\
\hline $2 s 3 d^{3} D_{1}$ & $2 s_{1 / 2} 3 d_{3 / 2}(1)$ & 106.2204 & -0.1649 & 0.0209 & -0.0880 & 105.9883 \\
\hline $2 p 3 p^{3} D_{2}$ & $2 p_{3 / 2} 3 p_{1 / 2}(2)$ & 89.8803 & -0.0835 & 0.0264 & -0.0067 & 89.8165 \\
\hline $2 p 3 p^{3} P_{2}$ & $2 p_{3 / 2} 3 p_{3 / 2}(2)$ & 91.0273 & -0.0802 & 0.0256 & -0.0051 & 90.9677 \\
\hline $2 p 3 p{ }^{1} D_{2}$ & $2 p_{1 / 2} 3 p_{3 / 2}(2)$ & 94.9325 & -0.0932 & 0.0259 & 0.0027 & 94.8679 \\
\hline $2 s 3 d^{3} D_{2}$ & $2 s_{1 / 2} 3 d_{3 / 2}(2)$ & 106.2738 & -0.1516 & 0.0196 & -0.0879 & 106.0539 \\
\hline $2 s 3 d^{1} D_{2}$ & $2 s_{1 / 2} 3 d_{5 / 2}(2)$ & 106.7656 & -0.1609 & 0.0202 & -0.0875 & 106.5376 \\
\hline $2 p 3 p^{3} D_{3}$ & $2 p_{3 / 2} 3 p_{3 / 2}(3)$ & 90.8176 & -0.0863 & 0.0255 & -0.0051 & 90.7518 \\
\hline $2 s 3 d^{3} D_{3}$ & $2 s_{1 / 2} 3 d_{5 / 2}(3)$ & 106.4321 & -0.0544 & -0.0033 & -0.0874 & 106.2871 \\
\hline $2 p 3 s^{3} P_{0}$ & $2 p_{1 / 2} 3 s_{1 / 2}(0)$ & 91.4073 & -0.0888 & 0.0273 & 0.0213 & 91.3670 \\
\hline $2 p 3 d^{3} P_{0}$ & $2 p_{3 / 2} 3 d_{3 / 2}(0)$ & 93.6979 & -0.1031 & 0.0279 & -0.0071 & 93.6156 \\
\hline $2 s 3 p^{3} P_{0}$ & $2 s_{1 / 2} 3 p_{1 / 2}(0)$ & 102.2052 & -0.1443 & 0.0208 & -0.0875 & 101.9942 \\
\hline $2 p 3 s^{3} P_{1}$ & $2 p_{3 / 2} 3 s_{1 / 2}(1)$ & 87.5287 & -0.0801 & 0.0262 & 0.0136 & 87.4884 \\
\hline $2 p 3 s{ }^{1} P_{1}$ & $2 p_{1 / 2} 3 s_{1 / 2}(1)$ & 91.4753 & -0.0904 & 0.0266 & 0.0213 & 91.4329 \\
\hline $2 p 3 d^{3} P_{1}$ & $2 p_{3 / 2} 3 d_{3 / 2}(1)$ & 93.8092 & -0.0939 & 0.0260 & -0.0070 & 93.7344 \\
\hline $2 p 3 d^{3} D_{1}$ & $2 p_{3 / 2} 3 d_{5 / 2}(1)$ & 94.8970 & -0.1000 & 0.0263 & -0.0062 & 94.8171 \\
\hline $2 p 3 d^{1} P_{1}$ & $2 p_{1 / 2} 3 d_{3 / 2}(1)$ & 98.4600 & -0.1067 & 0.0263 & 0.0003 & 98.3799 \\
\hline $2 s 3 p^{3} P_{1}$ & $2 s_{1 / 2} 3 p_{1 / 2}(1)$ & 102.2493 & -0.1383 & 0.0203 & -0.0873 & 102.0441 \\
\hline $2 s 3 p{ }^{1} P_{1}$ & $2 s_{1 / 2} 3 p_{3 / 2}(1)$ & 103.3370 & -0.1392 & 0.0195 & -0.0860 & 103.1313 \\
\hline $2 p 3 s{ }^{3} P_{2}$ & $2 p_{3 / 2} 3 s_{1 / 2}(2)$ & 87.3861 & -0.0802 & 0.0263 & 0.0136 & 87.3459 \\
\hline $2 p 3 d^{3} P_{2}$ & $2 p_{3 / 2} 3 d_{5 / 2}(2)$ & 94.0298 & -0.0948 & 0.0262 & -0.0068 & 93.9544 \\
\hline $2 p 3 d^{3} F_{2}$ & $2 p_{3 / 2} 3 d_{3 / 2}(2)$ & 94.1939 & -0.0960 & 0.0259 & -0.0064 & 94.1174 \\
\hline $2 p 3 d{ }^{1} D_{2}$ & $2 p_{1 / 2} 3 d_{3 / 2}(2)$ & 97.9568 & -0.1087 & 0.0264 & 0.0009 & 97.8754 \\
\hline $2 p 3 d^{3} D_{2}$ & $2 p_{1 / 2} 3 d_{5 / 2}(2)$ & 98.1817 & -0.1072 & 0.0260 & 0.0013 & 98.1018 \\
\hline $2 s 3 p^{3} P_{2}$ & $2 s_{1 / 2} 3 p_{3 / 2}(2)$ & 103.2248 & -0.1421 & 0.0196 & -0.0858 & 103.0165 \\
\hline $2 p 3 d^{3} F_{3}$ & $2 p_{3 / 2} 3 d_{3 / 2}(3)$ & 93.9594 & -0.0989 & 0.0255 & -0.0069 & 93.8790 \\
\hline $2 p 3 d^{3} D_{3}$ & $2 p_{3 / 2} 3 d_{5 / 2}(3)$ & 94.3546 & -0.0905 & 0.0256 & -0.0062 & 94.2835 \\
\hline $2 p 3 d^{1} F_{3}$ & $2 p_{1 / 2} 3 d_{5 / 2}(3)$ & 98.2537 & -0.1051 & 0.0260 & 0.0016 & 98.1761 \\
\hline $2 p 3 d^{3} F_{4}$ & $2 p_{3 / 2} 3 d_{5 / 2}(4)$ & 94.0797 & -0.0995 & 0.0251 & -0.0062 & 93.9990 \\
\hline
\end{tabular}

second-order Coulomb corrections and second-order BreitCoulomb corrections to reduced $E 1$ and $E 2$ matrix elements will be referred to as $Z^{(1)}, Z^{(2)}$, and $B^{(2)}$, respectively, throughout the text. These contributions are calculated in both length and velocity gauges. In this section, we show the importance of the different contributions, and discuss the gauge dependence of the $E 1$ and $E 2$ matrix elements.

In Fig. 3, differences between length and velocity forms are illustrated for the various contributions to uncoupled $0-2 p_{3 / 2} 3 d_{3 / 2}(1)$ matrix element, where 0 is the ground state. In the case of $E 1$ transitions, the first-order matrix element $Z^{(1)}$ is proportional to $1 / Z$, the second-order Coulomb matrix element $Z^{(2)}$ is proportional to $1 / Z^{2}$, and the second-order Breit-Coulomb matrix element $B^{(2)}$ is almost independent of $Z$ (see Ref. [37]) for high $Z$. Therefore, we plot $Z^{(1)} Z$,

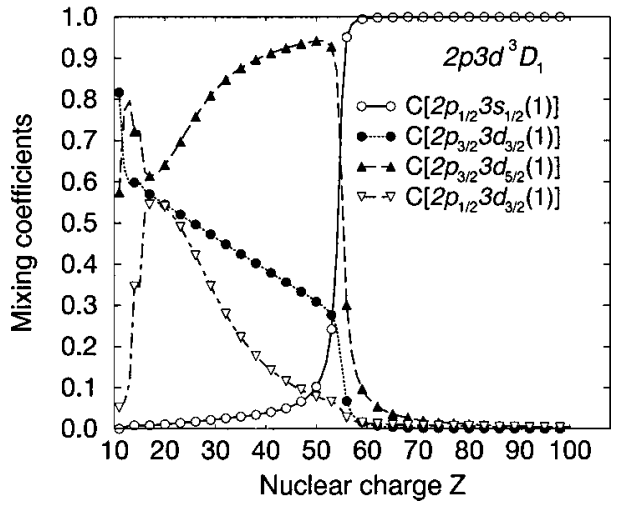

FIG. 1. Mixing coefficients for the $2 p 3 d^{3} D_{1}$ level as functions of $Z$. 


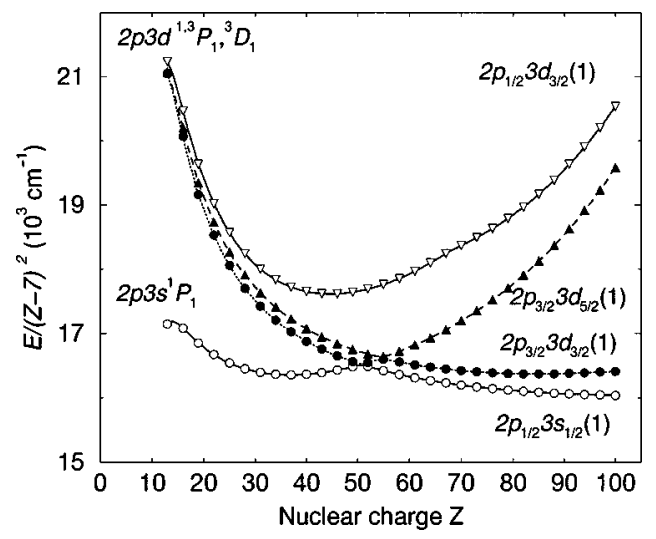

FIG. 2. Energies $\left[E /(Z-7)^{2}\right.$ in $\left.10^{3} \mathrm{~cm}^{-1}\right]$ of $2 l 3 l^{\prime}$ odd-parity states with $J=1$ as functions of $Z$.

$Z^{(2)} Z^{2}$, and $10^{4} B^{(2)}$ in Fig. 3. As one can see from Fig. 3, all these contributions are positive, except for the second-order matrix elements $Z^{(2)}$ in length form, which becomes negative for $Z>21$.

The difference between length and velocity forms for the $E 2$ uncoupled $0-2 p_{3 / 2} 3 p_{1 / 2}(2)$ matrix element is illustrated in Fig. 4. In the case of $E 2$ transitions, the first-order matrix element $Z^{(1)}$ is proportional to $1 / Z^{2}$, the second-order Coulomb matrix element $Z^{(2)}$ is proportional to $1 / Z^{3}$, and the second-order Breit-Coulomb matrix element $B^{(2)}$ is proportional to $1 / Z$ for high $Z$. Taking into account these dependencies, we plot $Z^{(1)} Z^{2}, Z^{(2)} Z^{3}$, and $10^{4} B^{(2)} Z$ in Fig. 4. The second-order Breit-Coulomb correction to the E2 matrix element $B^{(2)}$ is much smaller in velocity form than in length form, as seen in the figure.

The differences between results in length and velocity forms, shown in Figs. 3 and 4, are compensated by additional second-order terms called "derivative terms" $P^{\text {(derv) }}$; they are defined by Eq. (2.16) in Ref. [39]. The derivative terms arise because transition amplitudes depend on the energy, and the transition energy changes order by order in MBPT calculations.

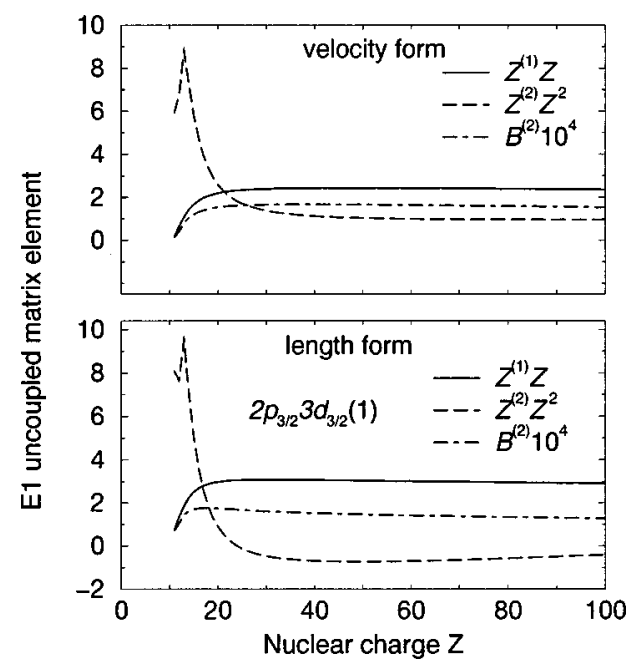

FIG. 3. Contributions to the $E 1$ uncoupled matrix element (a.u.) for the transition from the $2 p_{3 / 2} 3 d_{3 / 2}(1)$ state to the ground state calculated in length and velocity forms in Ne-like ions.

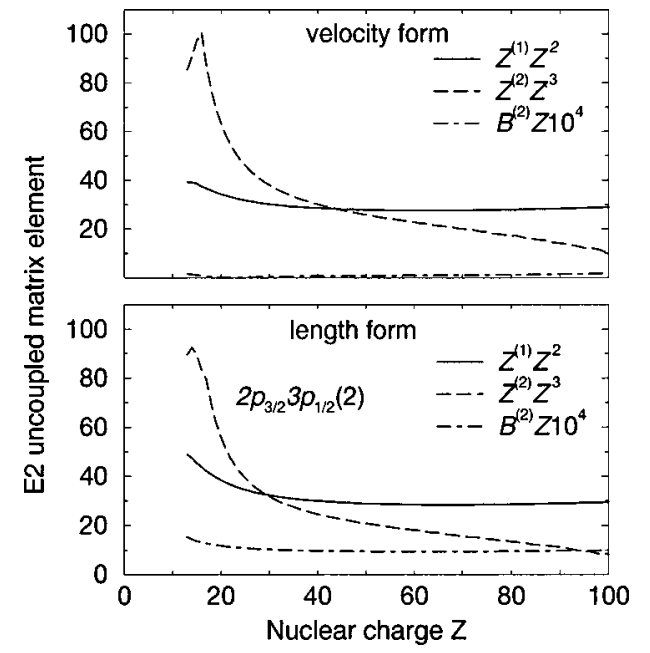

FIG. 4. Contributions to the $E 2$ uncoupled matrix element (a.u.) for the transition from the $2 p_{3 / 2} 3 p_{1 / 2}(1)$ state to the ground state calculated in length and velocity forms in Ne-like ions.

In Table $\mathrm{V}$, we list values of uncoupled first- and secondorder $E 1$ and $E 2$ matrix elements $Z^{(1)}, Z^{(2)}$, and $B^{(2)}$, together with derivative terms $P^{\text {(derv) }}$ for Ne-like molybdenum, $Z=42$. We list values for the seven $E 1$ transitions between odd-parity states with $J=1$ and the ground state and the five $E 2$ transitions between even-parity states with $J=2$ and the ground state, respectively. Matrix elements in both length $(L)$ and velocity $(V)$ forms are given. We can see that the firstorder matrix elements, $Z_{L}^{(1)}$ and $Z_{V}^{(1)}$, differ by $5-10 \%$; however, the $L-V$ differences between second-order matrix elements are much larger for some transitions. It can be also seen from Table $\mathrm{V}$ that for the $E 1$ transitions the derivative term in length form, $P_{L}^{(\text {derv })}$, is almost equal to $Z_{L}^{(1)}$ but the derivative term in velocity form, $P_{V}^{(\text {derv })}$, is smaller than $Z_{V}^{(1)}$ by 3-4 orders of magnitude. For the $E 2$ transition, the value of $P^{(\text {derv })}$ in velocity form is almost, equal to $Z^{(1)}$ in velocity form, and the $P^{(\text {derv })}$ in length form is larger by factor of 2 than $Z^{(1)}$ in length form.

Values of $E 1$ and E2 coupled reduced matrix elements in length and velocity forms are given in Table VI for the transitions considered in Table V. Although we use an intermediate-coupling scheme, it is nevertheless convenient to label the physical states using the $j j$ scheme. The first two columns in Table VI show $L$ and $V$ values of coupled reduced matrix elements calculated in first order. The $L-V$ difference is about $5-10 \%$. Including the second-order contributions (columns headed MBPT in Table VI) decreases the $L-V$ difference to $0.002-0.2 \%$. This extremely small $L-V$ difference arises because we start our MBPT calculations using a nonlocal Dirac-Fock (DF) potential. If we were to replace the DF potential by a local potential, the differences would disappear completely. It should be emphasized that we include the negative-energy state (NES) contributions to sums over intermediate states (see Ref. [37] for details). Neglecting the NES contributions leads to small changes in the $L$-form matrix elements but to substantial changes in some of the $V$-form matrix elements, with a consequent loss of gauge independence. 
TABLE V. Contributions to $E 1$ and $E 2$ uncoupled reduced matrix elements (a.u.) in length $L$ and velocity $V$ forms for transitions from $a v(J)$ states into the ground state in $\mathrm{Mo}^{32+}$.

\begin{tabular}{|c|c|c|c|c|c|c|c|c|}
\hline$a v(J)$ & $Z_{L}^{(1)}$ & $Z_{V}^{(1)}$ & $Z_{L}^{(2)}$ & $Z_{V}^{(2)}$ & $B_{L}^{(2)}$ & $B_{V}^{(2)}$ & $P_{L}^{(\text {derv })}$ & $P_{V}^{(\text {derv })}$ \\
\hline \multicolumn{9}{|c|}{$E 1$ uncoupled reduced matrix elements } \\
\hline $2 p_{3 / 2} 3 s_{1 / 2}(1)$ & -0.039269 & -0.037253 & -0.001920 & -0.001694 & -0.000093 & -0.000020 & -0.038945 & 0.000031 \\
\hline $2 p_{1 / 2} 3 s_{1 / 2}(1)$ & -0.024205 & -0.022987 & -0.001469 & -0.001262 & -0.000111 & -0.000048 & -0.023881 & 0.000214 \\
\hline $2 p_{3 / 2} 3 d_{3 / 2}(1)$ & 0.060681 & 0.057688 & -0.000580 & 0.000595 & 0.000101 & -0.000022 & 0.060625 & 0.000296 \\
\hline $2 p_{3 / 2} 3 d_{5 / 2}(1)$ & 0.181119 & 0.172191 & -0.001765 & 0.001839 & 0.000254 & -0.000065 & 0.179768 & -0.001475 \\
\hline $2 p_{1 / 2} 3 d_{3 / 2}(1)$ & -0.129576 & -0.123282 & 0.001442 & -0.001070 & -0.000281 & 0.000036 & -0.128916 & 0.000399 \\
\hline $2 s_{1 / 2} 3 p_{1 / 2}(1)$ & 0.055251 & 0.052692 & 0.000859 & 0.001187 & 0.000003 & -0.000069 & 0.054858 & -0.000027 \\
\hline $2 s_{1 / 2} 3 p_{3 / 2}(1)$ & 0.072600 & 0.069291 & 0.001306 & 0.001650 & 0.000066 & -0.000036 & 0.071730 & -0.000672 \\
\hline \multicolumn{9}{|c|}{ E2 uncoupled reduced matrix elements } \\
\hline $2 p_{3 / 2} 3 p_{1 / 2}(2)$ & 0.016820 & 0.016077 & 0.000319 & 0.000392 & 0.000023 & 0.000002 & 0.033582 & 0.016077 \\
\hline $2 p_{3 / 2} 3 p_{3 / 2}(2)$ & 0.016157 & 0.015372 & 0.000334 & 0.000405 & 0.000030 & 0.000008 & 0.032213 & 0.015372 \\
\hline $2 p_{1 / 2} 3 d_{3 / 2}(2)$ & 0.014794 & 0.014005 & 0.000345 & 0.000412 & 0.000044 & 0.000015 & 0.029449 & 0.014005 \\
\hline $2 s_{1 / 2} 3 d_{3 / 2}(2)$ & -0.028815 & -0.027526 & -0.000636 & -0.000962 & -0.000046 & -0.000012 & -0.057507 & -0.027526 \\
\hline $2 s_{1 / 2} 3 d_{5 / 2}(2)$ & -0.035170 & -0.033255 & -0.000878 & -0.001292 & -0.000047 & -0.000007 & -0.070016 & -0.033255 \\
\hline
\end{tabular}

\section{E. Magnetic-dipole and magnetic-quadrupole matrix elements}

We calculate magnetic-dipole (M1) matrix elements for the transitions between the six even-parity excited states $2 s_{1 / 2} 3 s_{1 / 2}(1), 2 s_{1 / 2} 3 d_{3 / 2}(1)$, and $2 p_{j} 3 p_{j^{\prime}}(1)$ and the ground state, and magnetic-quadrupole (M2) matrix elements for the transitions between the six odd-parity $2 s_{1 / 2} 3 p_{3 / 2}(2)$, $2 p_{3 / 2} 3 s_{1 / 2}(2)$, and $2 p_{j} 3 d_{j^{\prime}}(2)$ excited states and the ground state for Ne-like ions with nuclear charges $Z=11-100$. We calculate first- and second-order Coulomb corrections, second-order Breit-Coulomb corrections, and second-order derivative terms to reduced $M 1$ and $M 2$ matrix elements $Z^{(1)}, Z^{(2)}, B^{(2)}$, and $P^{(\text {derv })}$, respectively, using the method described in Ref. [39]. In this section, we illustrate the importance of the relativistic and frequency-dependent contributions to the first-order $M 1$ and $M 2$ matrix elements. We

TABLE VI. $E 1$ and $E 2$ coupled reduced matrix elements (a.u.) in length $L$ and velocity $V$ forms for transitions from $a v(J)$ states to the ground state in $\mathrm{Mo}^{32+}$.

\begin{tabular}{|c|c|c|c|c|}
\hline \multirow[b]{2}{*}{$a v(J)$} & \multicolumn{2}{|c|}{ First order } & \multicolumn{2}{|c|}{ MBPT } \\
\hline & $L$ & V & $L$ & $V$ \\
\hline \multicolumn{5}{|c|}{$E 1$ coupled reduced matrix elements } \\
\hline $2 p_{3 / 2} 3 s_{1 / 2}(1)$ & 0.046140 & 0.043791 & 0.048063 & 0.047973 \\
\hline $2 p_{1 / 2} 3 s_{1 / 2}(1)$ & -0.036248 & -0.034441 & -0.037756 & -0.037685 \\
\hline $2 p_{3 / 2} 3 d_{3 / 2}(1)$ & -0.004052 & -0.003851 & -0.003992 & -0.003992 \\
\hline $2 p_{3 / 2} 3 d_{5 / 2}(1)$ & 0.172035 & 0.163554 & 0.170333 & 0.170226 \\
\hline $2 p_{1 / 2} 3 d_{3 / 2}(1)$ & -0.157792 & -0.150125 & -0.156141 & -0.156054 \\
\hline $2 s_{1 / 2} 3 p_{1 / 2}(1)$ & -0.039214 & -0.037421 & -0.040111 & -0.040065 \\
\hline $2 s_{1 / 2} 3 p_{3 / 2}(1)$ & 0.065087 & 0.062157 & 0.066582 & 0.066509 \\
\hline \multicolumn{5}{|c|}{$E 2$ coupled reduced matrix elements } \\
\hline $2 p_{3 / 2} 3 p_{1 / 2}(2)$ & 0.017202 & 0.016422 & 0.016832 & 0.016846 \\
\hline $2 p_{3 / 2} 3 p_{3 / 2}(2)$ & -0.016568 & -0.015830 & -0.016269 & -0.016281 \\
\hline $2 p_{1 / 2} 3 d_{3 / 2}(2)$ & -0.015765 & -0.015074 & -0.015550 & -0.015560 \\
\hline $2 s_{1 / 2} 3 d_{3 / 2}(2)$ & 0.010784 & 0.010309 & 0.010632 & 0.010634 \\
\hline $2 s_{1 / 2} 3 d_{5 / 2}(2)$ & -0.043530 & -0.041617 & -0.043257 & -0.043255 \\
\hline
\end{tabular}

also show the importance of the taking into account the second-order MBPT contributions to $M 1$ and $M 2$ matrix elements, and we subsequently discuss the necessity of including the negative-energy contributions to sums over intermediate states.

The differences between first-order $M 1$ uncoupled matrix elements, calculated in nonrelativistic, relativistic frequencyindependent, and relativistic frequency-dependent approximations are illustrated for the $0-2 p_{1 / 2} 3 p_{1 / 2}(1)$ matrix element in Fig. 5. The corresponding matrix elements are labeled $Z^{(1)}(\mathrm{NR}), Z^{(1)}(\mathrm{R})$, and $Z^{(1)}(\mathrm{RF})$. Formulas for nonrelativistic and relativistic frequency-dependent first-order M1 matrix elements are given in Ref. [38]. We also plot the second-order Coulomb contributions, $Z^{(2)}$, and the secondorder Breit-Coulomb contributions, $B^{(2)}$, in the same figure. As we observe from Fig. 5, the values of $Z^{(1)}(\mathrm{NR})$ are twice as small as the values of $Z^{(1)}(\mathrm{R})$ and $Z^{(1)}(\mathrm{RF})$. Therefore, relativistic effects are very large for $M 1$ transitions. The frequency-dependent relativistic matrix elements $Z^{(1)}(\mathrm{RF})$ differ from the relativistic frequency-independent matrix elements $Z^{(1)}(\mathrm{R})$ by 5-10\%. The differences between other

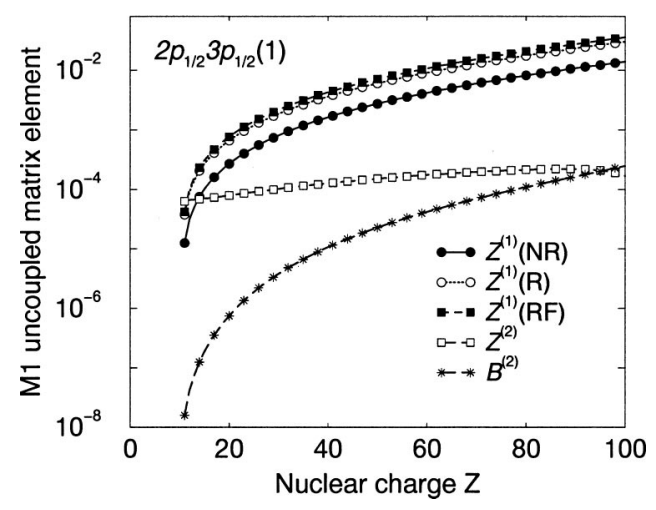

FIG. 5. Contributions to $M 1$ uncoupled matrix elements (a.u.) from the $2 p_{1 / 2} 3 p_{1 / 2}(1)$ state to the ground state in Ne-like ions as functions of $Z$. 


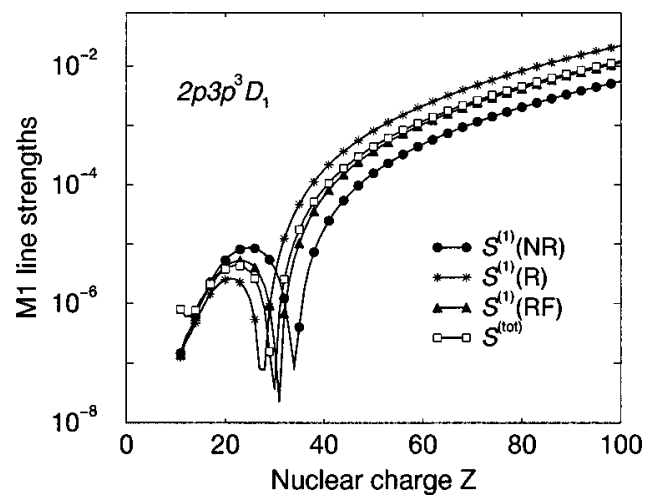

FIG. 6. Line strengths (a.u.) for magnetic-dipole transition from the $2 p 3 p{ }^{3} D_{1}$ state to the ground state in Ne-like ions as functions of $Z$.

first-order matrix elements calculated with and without frequency dependence are also on the order of a few percent. Uncoupled second-order $M 1$ matrix elements $Z^{(2)}$ are comparable to first-order matrix elements $Z^{(1)}(\mathrm{RF})$ for small $Z$ but the relative size of the second-order contribution decreases for high $Z$. This is expected since second-order Coulomb matrix elements $Z^{(2)}$ are proportional to $Z$ for high $Z$, while first-order matrix elements $Z^{(1)}(\mathrm{RF})$ grow as $Z^{2}$. The second-order Breit-Coulomb matrix elements $B^{(2)}$ are proportional to $Z^{3}$, and become comparable to $Z^{(2)}$ for very high $Z$.

In Fig. 6, we illustrate the $Z$ dependence of the line strengths of the $M 1$ transition from the $2 p 3 p^{3} D_{1}$ excited state to the ground state. In this figure, we plot the values of the first-order line strengths $S^{(1)}(\mathrm{NR}), S^{(1)}(\mathrm{R})$, and $S^{(1)}(\mathrm{RF})$ calculated in the same approximations as the $M 1$ uncoupled matrix elements: nonrelativistic, relativistic frequencyindependent, and relativistic frequency-dependent approximations, respectively. The total line strengths $S^{(\text {tot })}$, which include second-order corrections, are also plotted. Strong mixing inside of the even-parity complex with $J=1$, between $2 p_{3 / 2} 3 p_{1 / 2}$ and $2 p_{3 / 2} 3 p_{3 / 2}$ states occurring for small $Z$, leads to the sharp features in the line strengths seen in the graphs. The deep minimum in Fig. 6 shifts when different approximations are used for the calculation of line strengths. This shift in the placement of the minimum leads to difficulties in comparison of data for $M 1$ transitions obtained in nonrelativistic and relativistic approximations. We discuss this question further in Sec. III.

$A b$ initio relativistic calculations require a careful treatment of negative-energy states (virtual electron-positron pairs). In second-order matrix elements, such contributions explicitly arise from those terms in the sum over states for which $\varepsilon_{i}<-m c^{2}$. The effect of the NES contributions to M1 amplitudes has been studied recently in Ref. [38]. The NES contributions drastically change the second-order BreitCoulomb matrix elements $B^{(2)}$. However, the second-order Breit-Coulomb correction contributes only $2-5 \%$ to uncoupled $M 1$ matrix elements and, as a result, negativeenergy states change the total values of $M 1$ matrix elements by a few percent only.

In Table VII, we list values of uncoupled first- and second-order $M 2$ matrix elements $Z^{(1)}, Z^{(2)}$, and $B^{(2)}$, together with derivative terms $P^{\text {(derv) }}$, for Ne-like molybdenum, $Z=42$. We list values for the six $M 2$ transitions between odd-parity states with $J=2$ and the ground state. We note that the first-order $M 2$ matrix element $0-2 p_{3 / 2} 3 p_{3 / 2}(2)$ vanishes because the corresponding factor $\left(\kappa_{a}+\kappa_{v}\right)$, where $\kappa_{i}$ is a relativistic angular momentum quantum number of a state $i$, in the equation for $Z^{(1)}$ is equal to zero for this transition. Differences between first-order uncoupled $M 2$ matrix elements calculated with and without frequency dependence are shown in first two columns of Table VII labeled $Z^{(1)}(\mathrm{R})$ and $Z^{(1)}(\mathrm{RF})$. As one can see from Table VII, the difference between $Z^{(1)}(\mathrm{R})$ and $Z^{(1)}(\mathrm{RF})$ is about $1 \%$.

The importance of negative-energy states is illustrated for the second-order Breit-Coulomb matrix elements $B^{(2)}$. In Table VII, we compare the values of $B^{(2)}($ pos) and $B^{(2)}($ neg), calculated with positive and negative part of spectra, respectively. The ratio of $B^{(2)}\left(\right.$ neg) to $B^{(2)}(\operatorname{pos})$ is about $10 \%$.

In Table VIII, we present results of line strengths $S$ for $M 2$ lines in Ne-like ions. In this table, the values of $S$ for $Z=20-100$ in steps of 10 are given. The $S$ values are obtained in the intermediate-coupling scheme; nevertheless, both $L S$ and $j j$ designations are shown in this table. Among the six transitions presented in Table VIII, there is one transition with a zero first-order uncoupled matrix element, $0-2 p_{3 / 2} 3 p_{3 / 2}(2)$. The nonzero $S$ value of this transition results from the nonvanishing second-order contribution. It can be seen from Table VIII that the $S$ value of this transition is the smallest of the six values for high $Z$.

TABLE VII. Contributions to $M 2$ uncoupled reduced matrix elements (a.u.) for transitions from oddparity states with $J=2$ into the ground state in $\mathrm{Mo}^{32+}$.

\begin{tabular}{crrrrrr}
\hline \hline$a v(J)$ & \multicolumn{1}{c}{$Z^{(1)}(\mathrm{R})$} & \multicolumn{1}{c}{$Z^{(1)}(\mathrm{RF})$} & \multicolumn{1}{c}{$Z^{(2)}$} & \multicolumn{1}{c}{$B^{(2)}(\mathrm{pos})$} & $B^{(2)}(\mathrm{neg})$ & \multicolumn{1}{c}{$P^{(\text {derv })}$} \\
\hline $2 p_{3 / 2} 3 s_{1 / 2}(2)$ & -0.153944 & -0.152793 & -0.002071 & -0.000283 & -0.000027 & -0.303282 \\
$2 p_{3 / 2} 3 d_{5 / 2}(2)$ & 0.799284 & 0.796894 & 0.021865 & 0.001083 & 0.000034 & 1.589006 \\
$2 p_{3 / 2} 3 d_{3 / 2}(2)$ & 0.000000 & 0.000000 & -0.001750 & 0.000008 & 0.000030 & 0.000000 \\
$2 p_{1 / 2} 3 d_{3 / 2}(2)$ & -0.099421 & -0.099668 & -0.001187 & -0.000258 & 0.000010 & -0.199831 \\
$2 p_{1 / 2} 3 d_{5 / 2}(2)$ & -0.326342 & -0.325136 & -0.012417 & -0.000669 & 0.000025 & -0.647860 \\
$2 s_{1 / 2} 3 p_{3 / 2}(2)$ & 0.283552 & 0.281548 & 0.005763 & 0.000031 & 0.000027 & 0.559088 \\
\hline \hline
\end{tabular}


TABLE VIII. Line strengths (a.u.) for magnetic-quadrupole lines as functions of $Z$ in Ne-like ions.

\begin{tabular}{ccccccccccc}
\hline \hline$L S$ designations & $Z=20$ & $Z=30$ & $Z=40$ & $Z=50$ & $Z=60$ & $Z=70$ & $Z=80$ & $Z=90$ & $Z=100$ & $j j$ designations \\
\hline $2 p 3 s^{3} P_{2}$ & $1.96[-1]$ & $5.99[-2]$ & $2.93[-2]$ & $1.81[-2]$ & $1.31[-2]$ & $1.06[-2]$ & $9.40[-3]$ & $8.92[-3]$ & $8.96[-3]$ & $2 p_{3 / 2} 3 s_{1 / 2}(2)$ \\
$2 p 3 d^{3} P_{2}$ & $2.99[0]$ & $1.06[0]$ & $2.24[-1]$ & $1.97[-2]$ & $2.76[-3]$ & $5.83[-4]$ & $1.57[-4]$ & $4.96[-5]$ & $1.75[-5]$ & $2 p_{3 / 2} 3 d_{3 / 2}(2)$ \\
$2 p 3 d^{3} F_{2}$ & $3.28[-1]$ & $3.26[-1]$ & $5.02[-1]$ & $4.22[-1]$ & $2.91[-1]$ & $2.07[-1]$ & $1.53[-1]$ & $1.16[-1]$ & $9.02[-2]$ & $2 p_{3 / 2} 3 d_{5 / 2}(2)$ \\
$2 p 3 d^{3} D_{2}$ & $2.83[-1]$ & $5.00[-2]$ & $1.53[-2]$ & $7.45[-3]$ & $4.34[-3]$ & $2.74[-3]$ & $1.79[-3]$ & $1.17[-3]$ & $8.32[-4]$ & $2 p_{1 / 2} 3 d_{3 / 2}(2)$ \\
$2 s 3 d^{3} D_{2}$ & $1.05[-2]$ & $1.07[-1]$ & $9.39[-2]$ & $6.50[-2]$ & $4.39[-2]$ & $2.97[-2]$ & $2.02[-2]$ & $1.37[-2]$ & $8.52[-3]$ & $2 p_{1 / 2} 3 d_{5 / 2}(2)$ \\
$2 s 3 d^{1} D_{2}$ & $2.00[-1]$ & $9.71[-2]$ & $5.91[-2]$ & $3.83[-2]$ & $2.52[-2]$ & $1.65[-2]$ & $1.05[-2]$ & $5.67[-3]$ & $3.51[-3]$ & $2 s_{1 / 2} 3 p_{3 / 2}(2)$ \\
\hline \hline
\end{tabular}

\section{COMPARISON OF RESULTS WITH OTHER THEORY AND EXPERIMENT}

\section{A. Transition energies}

In Table IX, we compare our MBPT results for energies with experimental measurements from Ref. [21], and with theoretical results from Refs. $[19,20]$. We list only comparisons for ions with $Z=42,48,54$, and 60. A comparison of our results with data from Refs. [19-21] for all ions from $Z=39$ to 60 is available as supplementary data in Ref. [44]. Our results are seen to be in close agreement with theoretical calculations from Refs. [19,20], and with experimental measurements from Ref. [21]. In Refs. [19,20], all virtual orbitals were generated in the field of the hole state, i.e., in the $1 s^{2} 2 s^{2} 2 p^{5} V^{(N-1)}$ potential. The choice of such a potential is very convenient if only one hole state is considered, which was the case in Refs. [19,20], where only $2 p 3 l(J)$ states were investigated. The influence of the $2 s 3 l(J)$ states can be taken into account by summing all-order diagrams. In the present paper, the $V^{(N)}\left(1 s^{2} 2 s^{2} 2 p^{6}\right)$ potential, which allows one to treat $2 s 3 l(J)$ and $2 p 3 l(J)$ states simultaneously, is chosen. The calculations in Refs. $[19,20]$ included thirdorder corrections that are omitted in our work. However, third-order corrections are small for the high $Z$ considered in Table IX, and do not significantly affect the results for high$Z$ ions. It was shown in Refs. $[19,20]$ that the third-order hole-core Coulomb correlation energy $E^{(3+)}$ is about 0.1 a.u. for $\mathrm{Ne}$ I. This correction decreases rapidly with $Z$; the value of $E^{(3+)}$ is less than 0.007 a.u. for $\mathrm{Zr}^{30+}$.

Energies of $2 s 2 p^{6} 3 p^{1,3} P_{1}$ levels are given in Table $\mathrm{X}$ for selected high- $Z$ ions. Our results are compared with measurements from Ref. [21] and results for $\mathrm{Ag}^{37+}, \mathrm{Xe}^{44+}$, $\mathrm{Yb}^{60+}, \mathrm{Au}^{69+}$, and $\mathrm{Bi}^{73+}$ reported by Beiersdorfer and coworkers [27,29,30], Chandler et al. [32], and Dietrich et al. [33]. The experimental results for $\mathrm{Ag}^{37+}$ in Refs. [21,27] and $\mathrm{Xe}^{44+}$ in Refs. [21,29] disagree by 0.03-0.07 a.u.; our MBPT results lie between these two experimental measurements. A complete tabulation of our data for $2 s_{1 / 2} 3 p_{j}(1)$ energies for ions with $Z=39-94$ and a comparison of those energies with all available experimental results is given as supplementary data in Ref. [44].

Our MBPT data are compared with measurements from Beiersdorfer and co-workers in Refs. [27,29-31], Chandler et al. [32], and Dietrich et al. [33] in Table XI, and found to be in excellent agreement with experimental measurements.

TABLE IX. Energies (a.u.) for the $2 p 3 s$ (1) and $2 p 3 d$ (1) levels in Ne-like ions given relative to the ground state. Comparison of the present MBPT data (a) with theoretical results from Avgoustoglou and co-workers [19,20] (b), and with experimental data from Aglitskii et al. [21] (c).

\begin{tabular}{|c|c|c|c|c|c|}
\hline & $\begin{array}{c}2 p_{3 / 2} 3 s_{1 / 2}(1) \\
2 p 3 s{ }^{3} P_{1}\end{array}$ & $\begin{array}{c}2 p_{1 / 2} 3 s_{1 / 2}(1) \\
2 p 3 s{ }^{1} P_{1}\end{array}$ & $\begin{array}{c}2 p_{3 / 2} 3 d_{3 / 2}(1) \\
2 p 3 d^{3} P_{1}\end{array}$ & $\begin{array}{c}2 p_{3 / 2} 3 d_{5 / 2}(1) \\
2 p 3 d^{3} D_{1}\end{array}$ & $\begin{array}{c}2 p_{1 / 2} 3 d_{3 / 2}(1) \\
2 p 3 d{ }^{1} P_{1}\end{array}$ \\
\hline \multicolumn{6}{|c|}{$Z=42$} \\
\hline (a) & 87.4885 & 91.4330 & 93.7344 & 94.8171 & 98.3800 \\
\hline (b) & 87.4933 & 91.4384 & 93.7406 & 94.8167 & 98.3837 \\
\hline (c) & 87.506 & 91.441 & & 94.821 & 98.356 \\
\hline \multicolumn{6}{|c|}{$Z=48$} \\
\hline (a) & 118.9269 & 126.0972 & 127.0644 & 128.5289 & 135.0259 \\
\hline (b) & 118.9323 & 126.1035 & 127.0774 & 128.5219 & 135.0293 \\
\hline (c) & 118.933 & 126.155 & 126.963 & 128.489 & 134.978 \\
\hline \multicolumn{6}{|c|}{$Z=54$} \\
\hline (a) & 154.9060 & 166.9707 & 165.4626 & 167.5019 & 178.4834 \\
\hline (b) & 154.9124 & 166.9801 & 165.4835 & 167.4993 & 178.4917 \\
\hline (c) & 154.856 & 166.929 & 165.426 & 167.365 & 178.400 \\
\hline \multicolumn{6}{|c|}{$Z=60$} \\
\hline (a) & 195.2675 & 214.8141 & 208.9688 & 211.5009 & 229.3971 \\
\hline (b) & 195.2755 & 214.8249 & 208.9978 & 211.4833 & 229.4005 \\
\hline (c) & 195.174 & & & 211.432 & 229.215 \\
\hline
\end{tabular}


TABLE X. Energies (a.u.) of the $2 s_{1 / 2} 3 p_{j}$ (1) levels in Ne-like ions given relative to the ground state. Comparison of our MBPT results with experimental data.

\begin{tabular}{|c|c|c|c|c|}
\hline & $\begin{array}{c}2 s_{1 / 2} 3 p_{1 / 2}(1) \\
\text { MBPT }\end{array}$ & $\begin{array}{c}2 s_{1 / 2} 3 p_{1 / 2}(1) \\
\text { Expt. }\end{array}$ & $\begin{array}{c}2 s_{1 / 2} 3 p_{3 / 2}(1) \\
\text { МBPT }\end{array}$ & $\begin{array}{c}2 s_{1 / 2} 3 p_{3 / 2}(1) \\
\text { Expt. }\end{array}$ \\
\hline$Z=47$ & 132.1937 & $\begin{array}{l}132.198^{\mathrm{a}} \\
132.23^{\mathrm{b}}\end{array}$ & 133.983 & $\begin{array}{l}133.962^{\mathrm{a}} \\
133.99^{\mathrm{b}}\end{array}$ \\
\hline$Z=54$ & 181.8632 & $\begin{array}{l}181.824^{\mathrm{a}} \\
181.875^{\mathrm{c}}\end{array}$ & 185.194 & $\begin{array}{l}185.134^{\mathrm{a}} \\
185.209^{\mathrm{c}}\end{array}$ \\
\hline$Z=57$ & 205.9817 & & 210.231 & $\begin{array}{l}210.163^{\mathrm{a}} \\
210.25^{\mathrm{c}}\end{array}$ \\
\hline$Z=70$ & 332.1460 & & 343.264 & $343.29^{d}$ \\
\hline$Z=79$ & 443.7867 & $443.62^{\mathrm{e}}$ & 463.150 & $463.05^{\mathrm{c}}$ \\
\hline$Z=83$ & 500.8355 & $500.77^{\mathrm{f}}$ & 525.348 & \\
\hline
\end{tabular}

\begin{tabular}{ll}
\hline${ }^{\mathrm{a}}$ Aglitskii et al. [21]. & ${ }^{\mathrm{d}}$ Beiersdorfer et al. [30]. \\
${ }^{\mathrm{b}}$ Beiersdorfer et al. [27]. & ${ }^{\mathrm{e}}$ Chandler et al. [32]. \\
${ }^{\mathrm{c}}$ Beiersdorfer et al. [29]. & ${ }^{\mathrm{f}}$ Dietrich et al. [33].
\end{tabular}

Our theoretical data for other cases will be useful as benchmark data for future experiments. Results for the energies of all $362 l 3 l^{\prime}(J)$ levels for ions with $Z=47,54,57,60,63$, $70,79,83$, and 90 are available as supplementary data in Ref. [44].

\section{B. E1, E2, M1, and $M 2$ transition probabilities}

The $E 1, E 2, M 1$, and $M 2$ transition probabilities $A$ for the transitions between the ground state and $2 l j 3 l^{\prime} j^{\prime}(J)$ states are obtained using the relations

$$
\begin{aligned}
& A(E 1)=\frac{2.1420 \times 10^{10}}{(2 J+1)}(\Delta E)^{3} S(E 1) \mathrm{s}^{-1}, \\
& A(M 1)=\frac{2.8516 \times 10^{5}}{(2 J+1)}(\Delta E)^{3} S(M 1) \mathrm{s}^{-1}, \\
& A(E 2)=\frac{5.7032 \times 10^{4}}{(2 J+1)}(\Delta E)^{5} S(E 2) \mathrm{s}^{-1}, \\
& A(M 2)=\frac{7.5926 \times 10^{-1}}{(2 J+1)}(\Delta E)^{5} S(M 2) \mathrm{s}^{-1},
\end{aligned}
$$

where $\Delta E$ is the transition energy in atomic units and $S(E 1), S(M 1), S(E 2)$, and $S(M 2)$ are the corresponding line strengths in atomic units.

We present the resulting transition probabilities in Figs. 7-10. Transition rates for the seven $E 1$ lines $2 p 3 s^{3} P_{1},{ }^{1} P_{1}, 2 p 3 d^{3} P_{1},{ }^{3} D_{1},{ }^{1} P_{1}$ and $2 s 3 p^{3} P_{1},{ }^{1} P_{1}$

\begin{tabular}{|c|c|c|c|c|c|c|c|c|c|c|}
\hline $2 l 3 l^{\prime} L S J$ & $Z=47$ & $Z=54$ & $Z=57$ & $Z=60$ & $Z=63$ & $Z=70$ & $Z=79$ & $Z=83$ & $Z=90$ & $2 l j 3 l^{\prime} j^{\prime}(J)$ \\
\hline \multirow[t]{2}{*}{$2 p 3 s^{3} P_{1}$} & 113.37 & 154.91 & 174.55 & 195.27 & 217.03 & \multirow[t]{2}{*}{271.70} & 349.31 & 386.10 & 453.23 & \multirow[t]{2}{*}{$2 p_{3 / 2} 3 s_{1 / 2}(1)$} \\
\hline & $113.38^{\mathrm{a}}$ & $154.91^{\mathrm{b}}$ & $174.57^{\mathrm{b}}$ & $195.29^{\mathrm{b}}$ & $217.07^{\mathrm{b}}$ & & $349.36^{\mathrm{c}}$ & $386.15^{\mathrm{d}}$ & $453.44^{\mathrm{e}}$ & \\
\hline $2 p 3 s^{1} P_{1}$ & 119.89 & 165.46 & 186.58 & 208.97 & 232.64 & 292.90 & $\begin{array}{l}380.77 \\
380.65^{c}\end{array}$ & 423.61 & 504.24 & $2 p_{1 / 2} 3 s_{1 / 2}(1)$ \\
\hline \multirow{2}{*}{$2 p 3 d^{3} P_{1}$} & 121.16 & 166.97 & & 211.50 & & \multirow[t]{2}{*}{296.94} & $\begin{array}{l}380.65^{\mathrm{c}} \\
386.84\end{array}$ & & 514.03 & \multirow[t]{2}{*}{$2 p_{3 / 2} 3 d_{3 / 2}(1)$} \\
\hline & & $121.19^{\mathrm{a}}$ & $166.97^{\mathrm{b}}$ & $188.75^{\mathrm{b}}$ & $211.50^{\mathrm{b}}$ & & $386.59^{c}$ & $430.78^{\mathrm{d}}$ & & \\
\hline \multirow[t]{2}{*}{$2 p 3 d^{3} D_{1}$} & 122.55 & 167.50 & 190.10 & 214.81 & 241.38 & \multirow[t]{2}{*}{311.01} & 418.15 & 473.07 & 581.83 & \multirow[t]{2}{*}{$2 p_{3 / 2} 3 d_{5 / 2}(1)$} \\
\hline & $122.55^{\mathrm{a}}$ & $167.49^{\mathrm{b}}$ & $190.12^{\mathrm{b}}$ & $214.86^{\mathrm{b}}$ & & & $418.01^{\mathrm{c}}$ & $473.11^{\mathrm{d}}$ & & \\
\hline \multirow[t]{2}{*}{$2 p 3 d^{1} P_{1}$} & 128.46 & 178.48 & 202.96 & 229.40 & 257.89 & \multirow[t]{2}{*}{332.15} & 443.79 & 500.83 & 613.58 & \multirow[t]{2}{*}{$2 p_{1 / 2} 3 d_{3 / 2}(1)$} \\
\hline & $128.47^{\mathrm{a}}$ & $178.50^{\mathrm{b}}$ & & & & & $443.62^{\mathrm{c}}$ & $500.77^{\mathrm{d}}$ & & \\
\hline \multirow[t]{2}{*}{$2 s 3 p^{3} P_{1}$} & 132.19 & 181.86 & 205.98 & 231.90 & 259.72 & 333.42 & 450.80 & 511.78 & 634.10 & \multirow[t]{2}{*}{$2 s_{1 / 2} 3 p_{1 / 2}(1)$} \\
\hline & $132.23^{\mathrm{a}}$ & $181.87^{\mathrm{b}}$ & & & & $333.53^{f}$ & $450.58^{\mathrm{c}}$ & & & \\
\hline \multirow[t]{2}{*}{$2 s 3 p^{1} P_{1}$} & 133.98 & 185.19 & 210.23 & 237.26 & 266.39 & 343.26 & 463.15 & 525.35 & 649.99 & \multirow[t]{2}{*}{$2 s_{1 / 2} 3 p_{3 / 2}(1)$} \\
\hline & $133.99^{\mathrm{a}}$ & $185.21^{\mathrm{b}}$ & $210.25^{\mathrm{b}}$ & & & $343.2^{\mathrm{c}}$ & $463.05^{\mathrm{d}}$ & & & \\
\hline \multirow[t]{2}{*}{$2 p 3 s^{3} P_{2}$} & 113.20 & 154.71 & 174.34 & 195.04 & 216.78 & \multirow[t]{2}{*}{271.41} & 348.96 & 385.73 & 452.81 & \multirow[t]{2}{*}{$2 p_{3 / 2} 3 s_{1 / 2}(2)$} \\
\hline & $113.21^{\mathrm{a}}$ & $154.72^{\mathrm{b}}$ & $174.35^{\mathrm{b}}$ & $195.06^{\mathrm{b}}$ & $216.81^{\mathrm{b}}$ & & & & $453.03^{\mathrm{e}}$ & \\
\hline \multirow[t]{2}{*}{$2 p 3 p^{3} D_{2}$} & 116.08 & 158.20 & 178.10 & 199.09 & 221.13 & \multirow[t]{2}{*}{276.50} & 355.10 & 392.37 & 460.40 & \multirow[t]{2}{*}{$2 p_{3 / 2} 3 p_{1 / 2}(2)$} \\
\hline & $116.08^{\mathrm{a}}$ & $158.21^{\mathrm{b}}$ & $178.12^{\mathrm{b}}$ & & & & $355.24^{\mathrm{c}}$ & $393.52^{\mathrm{d}}$ & & \\
\hline \multirow[t]{2}{*}{$2 p 3 p^{3} P_{2}$} & 117.96 & 161.64 & 182.48 & 204.60 & 227.99 & \multirow[t]{2}{*}{287.58} & 374.57 & 417.02 & 496.94 & \multirow[t]{2}{*}{$2 p_{3 / 2} 3 p_{3 / 2}(2)$} \\
\hline & $117.96^{\mathrm{a}}$ & $161.65^{\mathrm{b}}$ & & & & & $374.48^{\mathrm{c}}$ & & & \\
\hline \multirow[t]{2}{*}{$2 p 3 p{ }^{1} D_{2}$} & 124.44 & 173.74 & 197.91 & 224.04 & 252.23 & \multirow[t]{2}{*}{326.77} & 443.28 & 503.83 & 625.38 & \multirow[t]{2}{*}{$2 p_{1 / 2} 3 p_{3 / 2}(2)$} \\
\hline & $124.45^{\mathrm{a}}$ & $173.77^{\mathrm{b}}$ & $197.93^{b}$ & & & & & & & \\
\hline \multirow[t]{2}{*}{$2 s 3 d^{1} D_{2}$} & 138.03 & 190.29 & 215.84 & 243.42 & 273.17 & 351.72 & 474.40 & 538.12 & 665.88 & $2 s_{1 / 2} 3 d_{5 / 2}(2)$ \\
\hline & & $190.32^{\mathrm{b}}$ & $215.86^{\mathrm{b}}$ & & & $351.73^{\mathrm{c}}$ & $474.42^{\mathrm{c}}$ & & & \\
\hline
\end{tabular}
are plotted in Fig. 7. The curves describing $2 p 3 s^{3} P_{1}$ and

TABLE XI. Energies (a.u.) of $2 l 3 l^{\prime}(J)$ levels in Ne-like ions given relative to the ground state. Comparison of our MBPT results with experimental data.

${ }^{\mathrm{a} B e i e r s d o r f e r}$ et al. [27].

${ }^{\mathrm{b}}$ Beiersdorfer et al. [29].

${ }^{\mathrm{c} C h a n d l e r}$ et al. [32].

\footnotetext{
${ }^{\mathrm{d}}$ Dietrich et al. [33].

${ }^{\mathrm{e}}$ Beiersdorfer et al. [31].

${ }^{\mathrm{f}}$ Beiersdorfer et al. [30].
} 


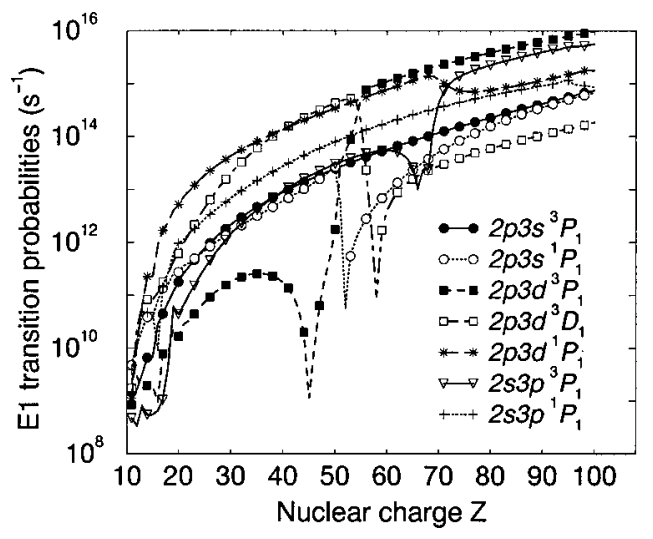

FIG. 7. $E 1$ transition probabilities $\left(s^{-1}\right)$ for Ne-like ions as functions of $Z$.

$2 s 3 p{ }^{1} P_{1}$ transition rates increase smoothly with $Z$ without any sharp features. However, the curves representing other transitions contain deep minima due to mixing of states. We already mentioned the strong mixing between $\left[2 p_{1 / 2} 3 s_{1 / 2}(1)\right]+\left[2 p_{3 / 2} 3 d_{3 / 2}(1)\right]+\left[2 p_{3 / 2} 3 d_{5 / 2}(1)\right]$ states in the interval $Z=51-55$. This mixing causes the exchange of $2 p 3 d^{3} P_{1}$ and $2 p 3 d^{3} D_{1}$ labels and the sharp minimum in the $2 p 3 s{ }^{1} P_{1}$ transition rate graph for $Z=52$. There are also minima in the curves describing the $2 p 3 d^{3} P_{1}(Z=45)$, $2 p 3 d^{3} D_{1}(Z=58)$, and $2 s 3 p^{3} P_{1}(Z=66)$ transition rates. We also compare our MBPT calculations with $E 1$ transition rates calculated by using the SUPERSTRUCTURE code in Refs. $[8,9,12]$ and the CIV3 code in Ref. [11]. All results agree at the level of 10-20\%, except for cases with singularities in the curves of transition rates. In these cases, (for example, the $2 p 3 d^{3} P_{1}$ transition rates for $Z=42$ ) our MBPT data are in better agreement with data from Ref. [5] than with data from Ref. [12].

Disagreement between our results and the theoretical calculations presented in Refs. $[8,9,12]$ is much larger for $M 1$ transition rates than for $E 1$ transition rates. This difference is explained by the large contribution of relativistic effects partly omitted in Refs. [8,9,12], but taken into account in our calculations. In Sec. II, we compared results for $M 1$ un-

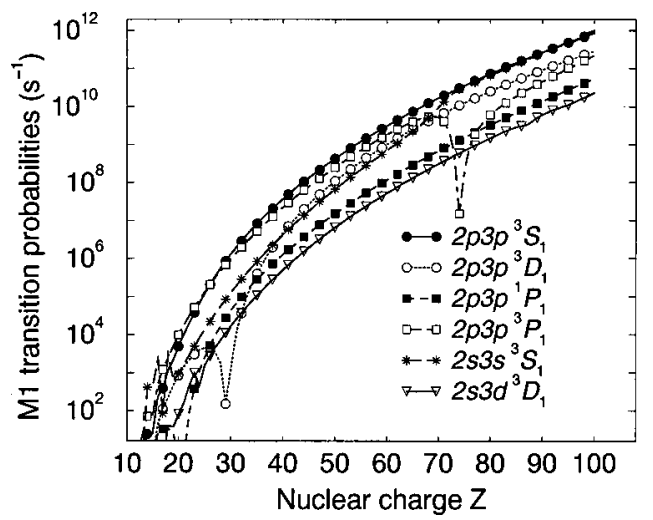

FIG. 8. $M 1$ transition probabilities $\left(s^{-1}\right)$ for Ne-like ions as functions of $Z$.

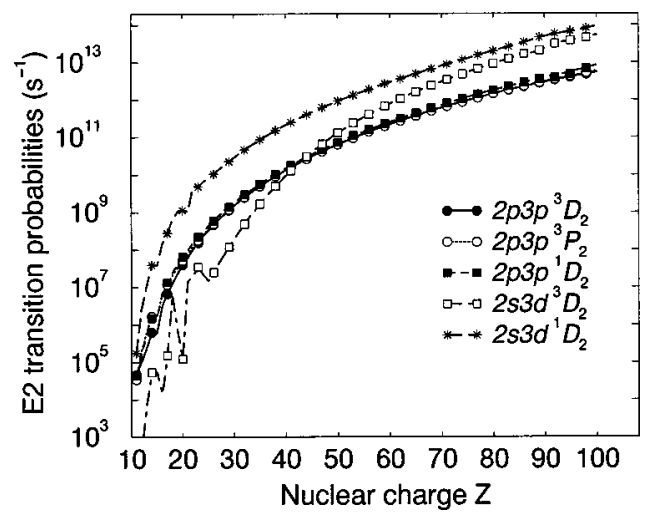

FIG. 9. $E 2$ transition probabilities $\left(s^{-1}\right)$ for Ne-like ions as functions of $Z$.

coupled matrix elements (see Fig. 5) and $M 1$ line strengths (see Fig. 6) calculated using nonrelativistic, relativistic, and frequency-dependent relativistic first-order matrix elements. The relativistic effects modify uncoupled matrix elements by a factor of 2 , and drastically change the Z-dependence of coupled matrix elements. In the SUPERSTRUCTURE code $[8,9,12]$, the wave functions were determined by diagonalizing the nonrelativistic Hamiltonian using orbitals calculated in a scaled Thomas-Fermi-Dirac-Amaldi potential. These nonrelativistic functions were used to calculate $M 1$ matrix elements. Our MBPT results and results from SUPERSTRUCTURE given in Refs. [8,9,12], differ by $20-30 \%$ for $2 p 3 p^{3} P_{1}$ transition rates and by a factor of $2-3$ for $2 p 3 p{ }^{3} S_{1},{ }^{3} D_{1}$, and ${ }^{1} P_{1}$ transition rates. Much better agreement was found between our results and results obtained in Ref. [5], where a relativistic approach was used. The differences with nonrelativistic calculations are especially large for the transition rates in the interval of $Z$ around the minima of line strength curves, such as those shown in Fig. 6. As we discussed in Sec. II E, the minima occur for different values of $Z$ in nonrelativistic and relativistic approximations. Since the transition rates are calculated using these line strengths, they change rapidly with $Z$ around the minima, leading to large disagreements between our relativistic calculations and the nonrelativistic calculations of Refs. [8,9,12]. For example, our result for $2 p 3 p^{3} D_{1}$ transition rates in $\mathrm{Cu}^{19+}$,

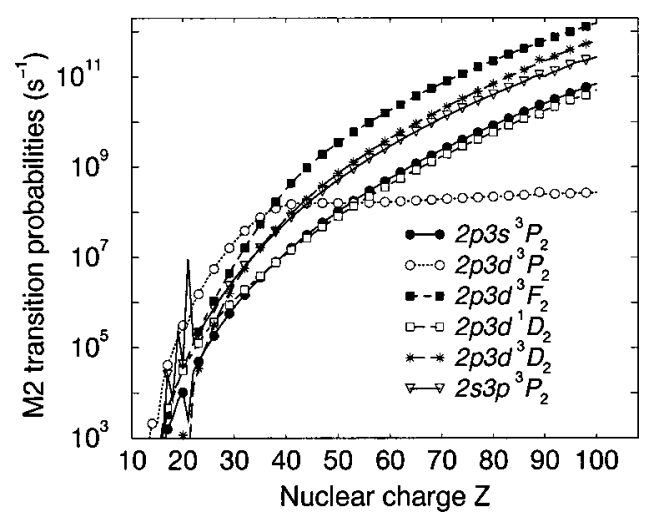

FIG. 10. $M 2$ transition probabilities $\left(s^{-1}\right)$ for Ne-like ions as functions of $Z$. 
where the minimum illustrated in Fig. 8 occurs, differs from the result of the SUPERSTRUCTURE code given in Ref. [12] by three orders of magnitude. The same minimum is seen in the curve describing the corresponding line strengths in Fig. 6.

The $E 2$ transition rates are less sensitive to relativistic effects than the $M 1$ transition rates. In Fig. 9, we show transition rates for the five $E 2$ lines, $2 p 3 p^{3} D_{2},{ }^{3} P_{2}$, and ${ }^{1} D_{2}$, and $2 s 3 d^{3} D_{2}$ and ${ }^{1} D_{2}$. It should be noted that there are the very small differences between the $2 p 3 p{ }^{3} D_{2},{ }^{3} P_{2}$, and ${ }^{1} D_{2}$ transition rates, which is seen from Fig. 9. All five $E 2$ transition rates increase smoothly with $Z$.

A similar smooth $Z$ dependence for $M 2$ rates is shown in Fig. 10. In this figure, the transition rates for six $M 2$ lines$2 p 3 s^{3} P_{2}, 2 p 3 d^{3} P_{2},{ }^{3} F_{2},{ }^{1} D_{2},{ }^{1} D_{2}$, and $2 s 3 p^{3} P_{2}$-are presented. The curve describing the $2 p 3 d^{3} P_{2}$ transition rate crosses the five curves describing other $M 2$ transitions rates and becomes the smallest one for $Z>54$. Such a decrease in the $2 p 3 d^{3} P_{2}$ transition rates can be explained by the fact that the $j j$ coupling scheme is more suitable than $L S$ coupling scheme for high $Z$. The value of uncoupled $0-2 p_{3 / 2} 3 d_{3 / 2}(2)$ matrix element is nonvanishing only because of the second-order contribution. Mixing inside the odd-parity complex with $J=2$ becomes less important with the increase of $Z$. The coupled $0-2 p 3 d^{3} P_{2}$ matrix element is approximately equal to the uncoupled $0-2 p_{3 / 2} 3 p_{3 / 2}(2)$ matrix element. This is a very interesting example of the case where intermediate coupling converts to pure $j j$ coupling.

Finally, we discuss some small singularities for low $Z$ in Figs. 7-10. These singularities are caused by the secondorder uncoupled matrix elements already discussed in Sec. II. The $2 s_{1 / 2} 3 s_{1 / 2}(1), 2 s_{1 / 2} 3 p_{j}(J)$, and $2 s_{1 / 2} 3 d_{j}(J)$ states are autoionizing for the $2 p_{j}$-hole threshold in the $Z$ $=11-20$ range. In this case, the singularity arises from the contribution of the continuous part of spectra in the sum over states for $Z^{(2)}$ (see Ref. [39]).

\section{CONCLUSION}

We have presented a systematic second-order relativistic MBPT study of excitation energies, reduced matrix elements, line strengths, and transition rates for $\Delta n=1$ electric- and magnetic-dipole and quadrupole transitions in Ne-like ions with nuclear charges $Z=11-100$. Our calculation of the retarded $E 1, E 2, M 1$, and $M 2$ matrix elements include correlation corrections from both Coulomb and Breit interactions. Contributions from virtual electron-positron pairs were also included in the second-order matrix elements. Both length and velocity forms of the $E 1$ and $E 2$ matrix elements were evaluated, and small differences, caused by the non-locality of the starting Hartree-Fock potential, were found between the two forms. Second-order MBPT transition energies were used to evaluate oscillator strengths and transition rates. Good agreement of our MBPT data with other accurate theoretical results leads us to conclude that the MBPT method provides accurate data for Ne-like ions. Results from the present calculations provide benchmark values for future theoretical and experimental studies of the neon isoelectronic sequence.

\section{ACKNOWLEDGMENTS}

The work of W.R.J. and M.S.S. was supported in part by National Science Foundation Grant No. PHY-99-70666. U.I.S. acknowledges partial support by Grant No. B503968 from Lawrence Livermore National Laboratory. U.I.S. would like to thank the members of the Data and Planning Center, the National Institute for Fusion Science for their hospitality, friendly support, and many interesting discussions.
[1] L.A. Bureeva and U.I. Safronova, Phys. Scr. 20, 81 (1979).

[2] E.V. Aglitskii, E.Ya. Golts, Yu.A. Levykin, A.M. Livshits, S.L. Mandelshtam, and A.S. Safronova, Opt. Spektrosk. 46, 1043 (1979) [Opt. Spectrosc. 46, 590 (1979)].

[3] U.I. Safronova, M.S. Safronova, and R. Bruch, Phys. Scr. 49, 446 (1994).

[4] E.P. Ivanova and A.V. Glushkov, J. Quant. Spectrosc. Radiat. Transf. 36, 127 (1986).

[5] E.P. Ivanova and A.V. Gulov, At. Data Nucl. Data Tables 49, 1 (1991).

[6] U.I. Safronova and J.-F. Wyart, Phys. Scr. 46, 134 (1992).

[7] E.P. Ivanova and I.P. Grant, J. Phys. B 31, 2671 (1998).

[8] A.K. Bhatia, U. Feldman, and J.F. Seely, At. Data Nucl. Data Tables 32, 435 (1985).

[9] A.K. Bhatia and G.A. Doschek, At. Data Nucl. Data Tables 52, 1 (1992).

[10] Y. Kagawa, Y. Honda, and S. Kiyokawa, Phys. Rev. A 44, 7092 (1991).

[11] A. Hibbert, M. Le Dourneuf, and M. Mohan, At. Data Nucl. Data Tables 53, 23 (1993).
[12] M. Cornille, J. Dubau, and S. Jacquemot, At. Data Nucl. Data Tables 58, 1 (1994).

[13] W. Fielder, Jr., D.L. Lin, and D. Ton-That, Phys. Rev. A 19, 741 (1979).

[14] A.K. Das, Astron. J. 468, 445 (1996).

[15] C. Hongshan, D. Chenzhong, and Z. Xiaoxin, J. Quant. Spectrosc. Radiat. Transf. 61, 143 (1999).

[16] A. Hibbert and M.P. Scott, J. Phys. B 27, 1315 (1994).

[17] P. Quinet, T. Gorilia, and E. Biémont, Phys. Scr. 44, 164 (1991).

[18] E. Avgoustoglou, W.R. Johnson, D.R. Plante, J. Sapirstein, S. Sheinerman, and S.A. Blundell, Phys. Rev. A 46, 5478 (1992).

[19] E. Avgoustoglou, W.R. Johnson, and J. Sapirstein, Phys. Rev. A 51, 1196 (1995).

[20] E. Avgoustoglou and Z.W. Liu, Phys. Rev. A 54, 1351 (1996).

[21] E.V. Aglitskii, E.P. Ivanova, S.A. Panin, U.I. Safronova, S.I. Ulitin, L.A. Vainshtein, and J.-F. Wyart, Phys. Scr. 40, 601 (1989).

[22] V.A. Boiko, A.Ya. Faenov, and S.A. Pikuz, J. Quant. Spectrosc. Radiat. Transf. 19, 11 (1979). 
[23] H. Gordon, M.G. Hobby, N.J. Peacock, and R.D. Cowan, J. Phys. B 12, 881 (1979).

[24] H. Gordon, H.G. Hobby, and N.J. Peacock, J. Phys. B 13, 1985 (1980).

[25] C. Jupén and U. Litzén, Phys. Scr. 30, 112 (1984).

[26] J.-C. Gauthier, J.-P. Geindre, P. Monier, E. Luc-Koenig, and J.-F. Wyart, J. Phys. B 19, L391 (1986).

[27] P. Beiersdorfer, M. Bitter, S. von Goeler, S. Cohen, K.W. Hill, J. Timberlake, R.S. Walling, M.H. Chen, P.L. Hagelstein, and J.H. Scofield, Phys. Rev. A 34, 1297 (1986).

[28] J.P. Buchet, M.C. Buchet-Poulizac, A. Denis, J. Desesquelles, M. Druetta, S. Martin, D. Leclerc, E. Luc-Koenig, and J.-F. Wyart, Nucl. Instrum. Methods Phys. Res. B 31, 177 (1988).

[29] P. Beiersdorfer, S. von Goeler, M. Bitter, E. Hinnov, R. Bell, S. Bernabei, J. Felt, K.W. Hill, R. Hulse, J. Stevens, S. Suckewer, J. Timberlake, A. Wouters, M.H. Chen, J.H. Scofield, D.D. Dietrich, M. Gerassimenko, E. Silver, R.S. Walling, and P.L. Hagelstein, Phys. Rev. A 37, 4153 (1988).

[30] P. Beiersdorfer, M.H. Chen, R.E. Marrs, and M.A. Levine, Phys. Rev. A 41, 3453 (1990).

[31] P. Beiersdorfer, Nucl. Instrum. Methods Phys. Res. B 56, 1144 (1991).

[32] G.A. Chandler, M.H. Chen, D.D. Dietrich, P.O. Egan, K.P. Ziock, P.H. Mokler, S. Reusch, and D.H.H. Hoffmann, Phys. Rev. A 39, 565 (1989).

[33] D.D. Dietrich, A. Simionovici, M.H. Chen, G. Chandler, C.J. Hailey, P.O. Egan, P.H. Mokler, S. Reusch, and D.H.H. Hoffmann, Phys. Rev. A 41, 1450 (1990).

[34] G. Yuan, Y. Kato, R. Kodama, K. Murai, and T. Kagava, Phys. Scr. 53, 197 (1996).

[35] N. Nakamura, D. Kato, and S. Ohtani, Phys. Rev. A 61, 052510 (2000).
[36] M.S. Safronova, W.R. Johnson, and U.I. Safronova, Phys. Rev. A 53, 4036 (1996).

[37] U.I. Safronova, W.R. Johnson, M.S. Safronova, and A. Derevianko, Phys. Scr. 59, 286 (1999).

[38] U.I. Safronova, W.R. Johnson, and A. Derevianko, Phys. Scr. 60, 46 (1999).

[39] U.I. Safronova, W.R. Johnson, and J.R. Albritton, Phys. Rev. A 62, 052505 (2000).

[40] W.R. Johnson, S.A. Blundell, and J. Sapirstein, Phys. Rev. A 37, 2764 (1988).

[41] M.H. Chen, K.T. Cheng, and W.R. Johnson, Phys. Rev. A 47, 3692 (1993).

[42] P.J. Mohr, Ann. Phys. (N.Y.) 88, 26 (1974); 88, 52 (1974); Phys. Rev. Lett. 34, 1050 (1975).

[43] W.R. Johnson, D.R. Plante, and J. Sapirstein, Adv. At., Mol., Opt. Phys. 35, 255 (1995).

[44] See EPAPS Document No. E-PLRAAN-64-114107 including three tables. Table I shows energies (a.u.) for the $2 p 3 s$ (1) and $2 p 3 d$ (1) levels in Ne-like ions relative to the ground state. A comparison of the MBPT data with theoretical results in Refs. $[19,20]$ and experimental data in Ref. [21] is given. In Table II, energies (a.u.) for the $2 s_{1 / 2} 3 p_{j}$ (1) levels in Ne-like ions are given relative to the ground state. A comparison of our MBPT results with experimental data in Refs. [21], [33], [32], [30], [27], and [29] is given. Table III shows energies (a.u.) for the $2 l 3 l^{\prime}(J)$ levels in Ne-like ions relative to the ground state. A comparison of our MBPT results with experimental data in Refs. [27], [29], [30], [32], [33], and [31] is given. This document may be retrieved via the EPAPS homepage (http:// www.aip.org/pubservs/epaps.html) or from ftp.aip.org in the directory /epaps/. See the EPAPS homepage for more information. 